\title{
Real-Time Optimizing Control of an Experimental Crosswind Power Kite
}

\author{
Sean Costello, Grégory François, and Dominique Bonvin
}

\begin{abstract}
The contribution of this article is to propose and experimentally validate an optimizing control strategy for power kites flying crosswind. The algorithm ensures the kite follows a reference path (control) and also periodically optimizes the reference path (efficiency optimization). The path-following part of the controller is capable of consistently following a reference path, despite significant time delays and wind variations, using position measurements only. The path-optimization part adjusts the reference path in order to maximize line tension. It uses a real-time optimization algorithm that combines off-line modeling knowledge and on-line measurements. The algorithm has been tested comprehensively on a small-scale prototype, and this article focuses on experimental results.
\end{abstract}

Index Terms-Path-following control, real-time optimization, airborne wind energy.

\section{INTRODUCTION}

W IND is one of the most promising renewable energy sources. Kite power is an emerging wind power technology with both great potential and great technical challenges. A global study based on experimental data estimated that the world's energy demand could be entirely satisfied using conventional wind turbines installed on only $2 \%$ of the world's land area [1]. However, in most locations the cost of wind energy is still significantly higher than that of energy produced from fossil sources, and wind power only accounts for about $2 \%$ of global electricity production. For wind power to be truly competitive, it needs to become significantly cheaper, more consistent and more efficient. "Airborne Wind Energy" using kites is a wind power concept that is radically different from conventional wind turbines. Kites are wings, ranging from flexible para-glider type designs to rigid composite aircraft wings, attached to the ground by a flexible tether. In addition to having low material costs, they can exploit the fact that wind strength and regularity increases with altitude [2], [3], [4]. Powerful kites already have wide application in sports: kite-propelled craft regularly break the world speed-sailing record. Now very large kites are being used to propel cargo ships [5], and many prototypes for electricity production are already in existence. An overview of this development can be found in [6], [7]. Although this initial development is

Dr. Costello (sean.c.costello@gmail.com) was with the Laboratoire d'Automatique, EPFL, Switzerland. He is currently with Leica Geosystems, St-Gallen, Switzerland.

Dr. François (gregory.francois@ed.ac.uk) was with the Laboratoire d'Automatique, EPFL, Switzerland. He is currently with the Institute of Material and Processes, School of Engineering, The University of Edinburgh, UK.

Prof. Bonvin (dominique.bonvin@epfl.ch) is with the Laboratoire d'Automatique, EPFL, Switzerland. encouraging, making the step to commercial power production requires great technical barriers to be overcome. These include finding kite and tether designs that can withstand long-term outdoor exposure and repetitive high forces while remaining light, seamless autonomous take-off and landing, and perfectly reliable autonomous operation in all conditions.

The control of kites is one of the most fundamental challenges to be tackled. A dynamically flying power kite is a fast, unstable system influenced by unpredictable wind disturbances, and usually only noisy and incomplete measurements are available. It is a testament to the difficulty of stabilizing a kite during dynamic flight that the first successful account of experimental kite control was published in 2013 [5], 33 years after research on kite power began [8]. Typically, in order to extract maximum power from the wind, a power kite is flown almost perpendicular to the wind, similarly to the blades of a wind turbine, reaching speeds many times that of the wind itself (easily in excess of $150 \mathrm{~km} / \mathrm{h}$ ). This is known as crosswind flight, and is the focus of this paper. Note that there are other useful modes of flight, such as static flight at the zenith, which are not treated here. During crosswind flight, if the kite is not constantly steered, it will crash in a matter of seconds. Hence, an "autopilot" must keep the kite flying in a wide variety of wind conditions, providing stability. What is more, unlike the blades of a wind turbine which must move in a circle, a kite can follow many different flight paths. The path the kite flies determines how much power is produced. Hence, in addition to keeping the kite from crashing, the autopilot must ensure the kite follows a path that is efficient for power production.

The field of kite control is young, yet varied. In theory, state-of-the-art Nonlinear Model Predictive Control (NMPC) is the perfect solution [9], [10], [11], given the complex nature of the control problem, the presence of operational constraints, and the necessity to optimize the kite's flight path. Unfortunately, this has yet to become a practical reality, mainly due to the inaccuracy of existing kite models, as NMPC relies on the quality of the model at hand. Many power kites are of the flexible kind, and accurate models [12], [13] are very complex, generally unsuitable for NMPC. We note, however, that with recent advances in MPC [14], this situation will hopefully change. There are, on the other hand, several accounts of experimentally validated geometric control laws. For example, [15] observed that a simple control scheme should aspire to control the kite's direction of motion, referred to as the velocity angle, which will also be used extensively in this paper. They combined an online system-identification algorithm with a Lyapunov-based control law. The control 
law attempts to choose the kite's velocity angle such that it smoothly attains the prescribed target trajectory. An additional contribution was to elegantly exploit the concept of geodesic curvature to simplify the problem of tracking on a sphere. Also, [5] developed a simple, robust cascade controller for kites, which was tested by years of sea trials on large vessels. Essentially, a low-level proportional controller regulates the kite's orientation (i.e. the direction the kite is pointing), while a higher-level guidance controller chooses the bang-bang reference orientation signal, based on the kite's current position. This results in a horizontal figure-of-eight pattern, which is generally considered to be the most efficient type of path for extracting energy from the wind. The resulting controller has only a few tuning parameters, however the effect of these parameters on the kite's trajectory is difficult to determine a priori. A similar cascade-control strategy was proposed and experimentally validated on a small prototype by [16]. The primary controlled variable was the kite's velocity angle in this case, which was again regulated by a simple low-level linear controller. The guidance strategy alternately directs the kite towards one of two points, producing the classic figure-ofeight pattern. The tuning parameters in this case can be used to choose the height, width and inclination of the figure-of-eight in an intuitive manner. The same authors extended this control law to also handle the retraction phase for a pumping-cycle generator, and successfully implemented the algorithm on a power-producing prototype [17]. Building on the work by [15], [18] proposed a more advanced path-following controller using a nonlinear guidance-law and successfully tested it on a 20$\mathrm{kW}$ pumping-cycle prototype. Feedback linearization is used to design a low-level velocity-angle controller. The guidance law aims to minimize the cross-track error, taking into account the kite's current velocity angle, the path's direction, and the curvature of the path. Successful implementation of a pathfollowing controller is also reported in [19], this time via waypoint tracking for rigid wings.

While several control solutions for kites now exist, and the most advanced of these are even capable of tracking relatively arbitrary paths, the path-planning problem during crosswind flight is still an open issue. Intelligent path planning is important because, although the kite is free to follow almost any flight path, it is the flight path that directly determines the aerodynamic force the kite experiences, and hence the power generated. Experimental studies have confirmed that the path taken by the kite significantly affects the power it can generate [20]. The path-planning problem results in an interesting optimal control problem that has been studied by a number of authors [9], [21], [22], [23], [24], [25]. However, despite promising recent work [26], there remains a gap between the theory and the application. Recently, more detailed models have been employed [27], particularly for rigid wings for which modeling is easier. Flexible power kites are very popular, and for these kites modeling is still quite approximate. In addition, effects such as wind gradients vary from location to location and cannot be known in advance. Hence, with the tools that are currently available, it remains questionable whether a purely model-based approach can calculate optimal paths for a real flexible kite. This is probably the reason why the most prominent available experimental study uses an experimental approach to tune the path the kite follows: [20], [28] proposed an algorithm that adjusts the height and lateral position of the kite's path in real time, using experimental data only. These two parameters are then optimized online using a gradient-search algorithm. Since the use of offline modeling to design the real-time optimization algorithm, and online experimental data to perform the optimization, has yielded promising results, this paper will develop a similar approach.

The contribution of this article, which presents a part of the work in [29], is to propose a combined control and optimization strategy for power kites flying crosswind, with a focus on experimental results (in fact, all data presented here is experimental). The control strategy provides both path control and path optimization. The entire algorithm is the result of many years of experimental work, and it has been incrementally developed with regular experiments to test each addition to the algorithm. A preliminary simulation study was presented in [30], and we now extend the presentation and give experimental results. The path-following part of the controller is capable of consistently following a wide variety of paths, despite significant time delays, using position measurements only. The path-optimization part maximizes line tension (efficiency) by combining offline modeling knowledge and online measurements. The online data ensures it can react to wind or system variations to maximize efficiency in different conditions. An additional small, but useful, contribution is to give some experimental data supporting a recently proposed modeling relationship for power kites, linking the decrease in the kite's lift-to-drag ratio to the steering deflection.

The paper is structured as follows. Section II describes the experimental setup. Section III develops a low-dimensional dynamic model of the system using existing models from the literature as a starting point, and validates the model using experimental data. Section IV presents a path-following controller for kites and analyzes its experimental performance. Section V presents the Real-Time Optimization (RTO) algorithm, describes how the effect of noise is mitigated, and illustrates its experimental performance.

\section{EXPERIMENTAL SETUP}

The experimental setups used in this work are small $(2.5$ and $\left.3.5-\mathrm{m}^{2}\right)$ kites on a short $(35 \mathrm{~m})$, fixed-length line, sensed and actuated from the ground by a mechanized station. This small-scale setup has the advantage of providing a relatively controlled environment, while retaining many of the properties of the much larger kites used in commercial settings, which typically use line lengths of 50-500 $\mathrm{m}$ and kite sizes of 10$300 \mathrm{~m}^{2} .^{1}$ Firstly, short lines experience negligible line drag during crosswind flight. This allows the kite's position to be quite precisely measured (to within several $\mathrm{cm}$ ) using line angles, and the attitude and the steering deflection to be precisely controlled. Secondly, short lines keep the kite close to the ground, that is, where the wind speed and direction are

\footnotetext{
${ }^{1}$ The Reynolds number of both a 3- $\mathrm{m}^{2}$ kite and a scaled-up 300- $\mathrm{m}^{2}$ kite in crosswind flight ensures turbulent flow [25], thus there are unlikely to be significant differences between their aerodynamic properties, and hence their behavior.
} 


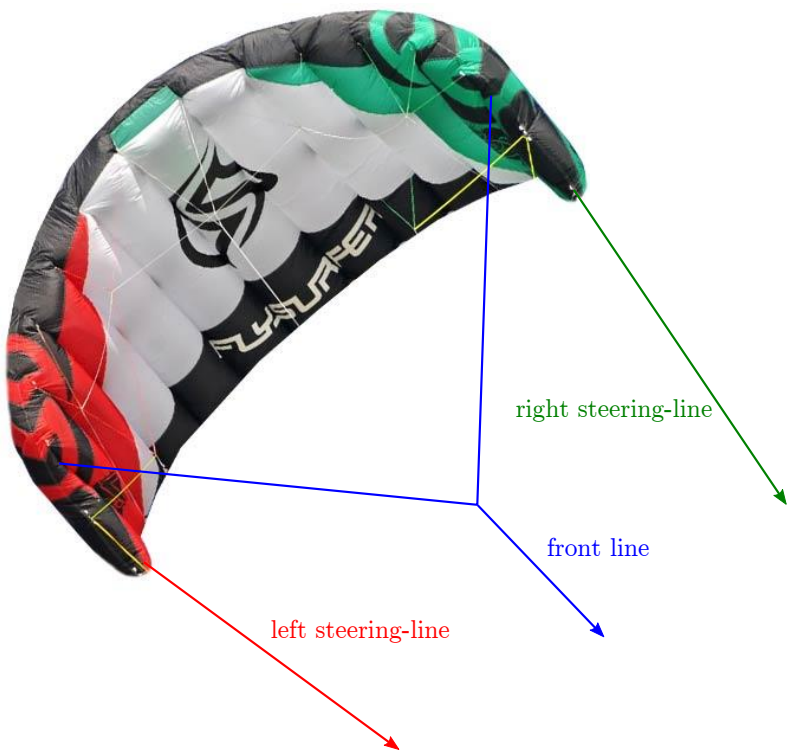

Fig. 1. The $2.5-\mathrm{m}^{2}$ Flysurfer Viron power kite used in this study.

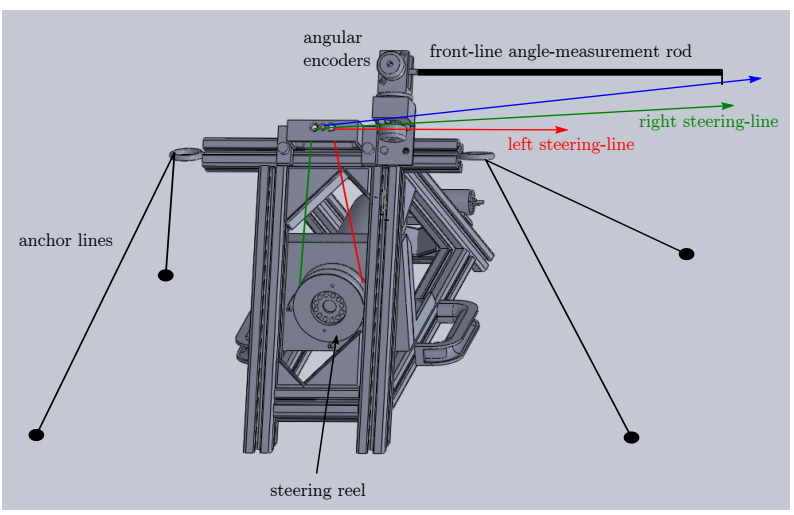

Fig. 2. The ground station.

measured. Hence, the small-scale system provides a controlled environment for validating modeling hypotheses and control strategies.

\section{A. Physical System}

The kites employed, one of which is shown in Figure 1, have the characteristics of standard power kites. Two commercial power kites were used: A $2.5-\mathrm{m}^{2}$ Flysurfer Viron and a 3.5$\mathrm{m}^{2}$ HQ Apex. Both are three-line $e^{2}$ kites; one front line takes about $90 \%$ of the force generated by the kite, the two lightlytensioned rear lines allow the kite to be maneuvered. There are two degrees of freedom to operate the kite: (i) adjusting the difference between the lengths of the rear lines allows the kite to be steered left or right, and (ii) adjusting the length of both rear lines relative to the front line allows some control over the kite's velocity by changing its angle-of-attack to the onrushing air. In this article, only the steering degree of freedom is adjusted automatically - the rear line lengths vary, but the

\footnotetext{
${ }^{2}$ This type of kite is often termed a 4-line kite also, as there are 4 attachment points on the kite. However, the two front lines join together, and there are only 3 attachment points on the control bar.
}

sum of their lengths is constant. The length of the front line is maintained constant throughout each experiment.

The geometry of the ground station, shown in Figure 2, has an important impact on the experiments. The lines coming from the kite are led through three small eyes placed closely together. This ensures that, as the kite moves around, and hence the angle of the lines changes, the relative lengths of the lines will not vary. The front line is attached to a load cell that measures the line tension. The angle of the front line between the station and the kite is measured by a 1-m long light carbonfiber rod with a small ring at the end, through which the front line passes. The angle of the rod, from which the line angle can be inferred, is measured by rotary encoders. The rear (steering) lines are wound in opposite directions around a reel, which is turned by a responsive and powerful servomotor. Following a change in the reference position, the motor has a settling time of about $30 \mathrm{~ms}$ in moderate winds. Rotating the reel shortens one line, while lengthening the other, achieving a steering effect. A high-precision ultrasonic anemometer mounted on a 3-m pole measures the wind speed and direction. The control algorithm runs in real time on a laptop with a sampling period of $30 \mathrm{~ms}$. Hence, accurate measurements of the the kite's position, the front-line tension and the wind speed and direction are available at the ground station, where the steering input is manipulated.

\section{B. Testing Conditions}

Wind speed is the most obvious testing condition that influences the kite's behavior. It affects the speed of the kite, the line tension it produces, and the kite's turning characteristics. Of equal importance, although less obvious, is the short-term variability of the wind's speed and direction, i.e. the gustiness of the wind, which is mostly decided by the ruggedness of the local terrain.

Tests were performed in locations with very different levels of gustiness, from (not very gusty) beaches to (very gusty) mountain ridges and narrow valleys. Very gusty conditions severely complicate modeling, but on the other hand, controllers should demonstrate robustness to a reasonable level of gustiness. The majority of the results presented here were obtained in (medium gusty) flat fields, with no obstructions to windward for at least 500 meters, using the $3.5 \mathrm{~m}^{2} \mathrm{HQ}$ Apex kite. The wind speed was $4-5 \mathrm{~m} \cdot \mathrm{s}^{-1}$, which is a gentle breeze. For any results obtained under different conditions, the wind speed and kite model are indicated in the figure caption.

\section{Modeling}

This section focuses on low-state-dimension dynamic models for flexible power kites. Much progress has been made in modeling the dynamics of flexible kites during the last decade [9], [21], [31], [32], [33], [13], [34], [12], [35], [36]. Relatively simple tendency models have between 3 and 10 states, while more complex models aimed at achieving great precision have up to several hundred states. The complex models are constructed afresh for each kite geometry, and are generally used in a simulation environment to validate control strategies. For the purposes of this article, tendency models are 


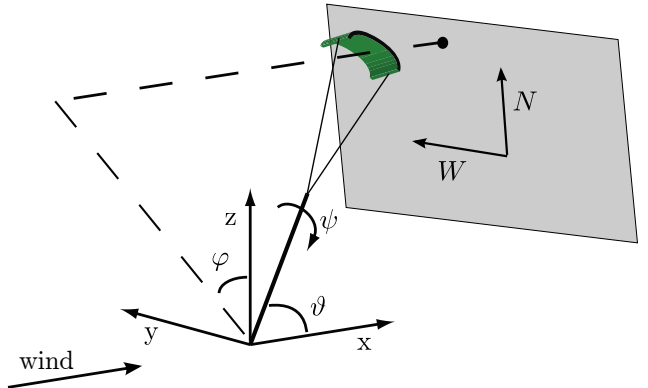

Fig. 3. Spherical coordinate system for the kite position. The $\mathrm{x}$ axis is aligned with the horizontal wind component, while the $\mathrm{z}$-axis is vertical and points upwards. $\varphi$ is the angle between the kite's position projected onto the $z-y$ plane and the $\mathrm{z}$ axis. $\vartheta$ is the angle between the kite's position and the $\mathrm{x}$ axis. The origin is at the point where the kite tether enters the ground station.

more useful, since they incorporate general characteristics that are reproduced by most flexible power kites and, through their simplicity, allow interesting insights into the kite's dynamic behavior. The two tendency models that are typically used for controller design and path optimization are the wellestablished point-mass model [9], [16], and that proposed by [5], henceforth referred to as the Erhard Model. Both models are general enough to apply to both ram-air kites and tube kites. If appropriate parameters are used, both models will predict qualitatively similar behavior. However, the Erhard Model is simpler and more intuitive, as it has only two aerodynamic parameters that can be calculated for a real system using straightforward experiments. It has also been successfully used to design a control algorithm for very large, commercial kites. Hence, as one of the simplest and bestvalidated flexible kite models, the Erhard Model is the starting point for understanding the experimental system in this paper. First, the coordinate system that will be used throughout the paper is defined. Next, the Erhard Model is described, and from this a second model based on the kite's velocity angle is derived. Finally, an addition to the turning law is derived from experimental data.

\section{A. Coordinate System}

The right-hand inertial frame used is depicted in Figure 3. As in [5], the spherical co-ordinates are judiciously chosen such that the polar angle, $\vartheta$, is measured from the axis aligned with the wind direction - this renders some expressions in the dynamic equations more concise, as the kite's behavior is symmetric about this axis (if, as we shall see is the case, the effect of gravity is neglected). The kite's position in Cartesian coordinates is given by:

$$
\mathbf{p}=r\left[\begin{array}{c}
\cos \vartheta \\
\sin \vartheta \sin \varphi \\
\sin \vartheta \cos \varphi
\end{array}\right],
$$

where $r$ is the (constant) length of the kite's tether, and $\vartheta$ and $\varphi$ are spherical coordinates for the kite's position.

The path-following control algorithm presented in this article uses the kite's position represented as a projection onto the $\{N, W\}$ plane shown in Figure 3 . The plane is defined by the two orthogonal vectors $\hat{\mathbf{e}}_{W}=\left[\begin{array}{lll}0 & 1 & 0\end{array}\right]^{T}$ and
$\hat{\mathbf{e}}_{N}=\left[\begin{array}{lll}-\sin \bar{\vartheta} & 0 & \cos \bar{\vartheta}\end{array}\right]^{T}$, which are tangent to the sphere upon which the kite can move at the point $\{\vartheta, \varphi\}=\{\bar{\vartheta}, 0\}$. The value of $\bar{\vartheta}$, which decides the region in which the $\{N, W\}$ plane is a good approximation of the sphere, is not fixed; it is chosen to suit the reference path to be followed.

Finally, we will also define the wind model employed in this work. A number of different wind-shear models exist to describe the variation of wind speed with altitude. We use the power law [3], which is one of the most common. The wind speed at the kite's current altitude, $w$, is given by:

$$
w=w_{\text {ref }}\left(z / z_{\text {ref }}\right)^{a},
$$

where $a$ is the surface friction coefficient, $w_{\text {ref }}$ is the reference wind speed at the reference altitude $z_{\text {ref }}$, and $z$ is the kite altitude.

\section{B. Erhard Model}

The Erhard Model [5] is surprisingly compact and yet encompasses the aspects of basic kite dynamics that are important for crosswind control. The important simplifying assumption made by the basic Erhard Model (the authors also proposed extensions that account for gravity [33]) is that both gravity and the kite's inertia are negligible ${ }^{3}$. For flexible kites, which are extremely light, this is a reasonable assumption during crosswind flight, during which the aerodynamic forces are typically at least one order of magnitude greater than gravitational and inertial forces. This reduces the number of states compared with the point-mass model, as the differential model equations then contain no acceleration terms. The dynamic equations for the model are:

$$
\begin{aligned}
& \dot{\vartheta}=\frac{w_{\mathrm{ap}}}{r}\left(\cos \psi-\frac{\tan \vartheta}{E}\right), \\
& \dot{\varphi}=-\frac{w_{\mathrm{ap}}}{r \sin \vartheta} \sin \psi, \\
& \dot{\psi}=w_{\mathrm{ap}} g_{\mathrm{s}} \delta+\dot{\varphi} \cos \vartheta,
\end{aligned}
$$

where $\psi$ is the kite orientation, $g_{\mathrm{s}}$ is the turning constant, and $E$ is the kite's lift-to-drag ratio. The steering deflection, $\delta$, is the system's manipulated variable. $w_{\mathrm{ap}}$ is the magnitude of the apparent wind projected onto the plane that is normal to $\mathbf{p}$, and is given by:

$$
w_{\mathrm{ap}}=w E \cos \vartheta
$$

Finally, the line tension is given by ${ }^{4}$ :

$$
T=\left(\frac{1}{2} \rho A w^{2}\right) E^{2}\left(1+\frac{1}{E^{2}}\right)^{\frac{3}{2}} \cos ^{2} \vartheta .
$$

Hence, using only three states representing position and orientation, the Erhard Model describes the kite's velocity, turning behavior and line tension.

\footnotetext{
${ }^{3}$ It could be argued that the empirical steering law accounts for the kite's rotational inertia, however inertial forces due to linear acceleration are certainly neglected.

${ }^{4}$ This expression assumes the kite operates with a lift coefficient of 1 , which is only approximately true. However, the assumption only introduces a proportional error that does not affect the algorithms in this article.
} 


\section{Cart Model}

The Erhard Model is remarkably straightforward, but it can be cast into an even simpler form that is more useful when sensors are not mounted on the kite. We named this the Cart Model in [29], due to its resemblance to a simple cart-like mobile robot. A similar model was presented by [37], who call it a Unicycle Model. The difference between our presentation and that in [37] is that we derive the Cart Model from the Erhard Model, which clarifies the simplifying assumptions. Also, the model obtained in this way is more detailed, as explicit expressions for the kite's speed and steering dynamics are obtained.

The main idea is to use an alternative state variable, the velocity angle, which was introduced and used for kite control by [16], [18]. While the kite's position is generally measured on all experimental systems, an accurate measurement of the kite's orientation, the third state in the Erhard Model, is not always available. This is certainly the case for our experimental setup, which has no Inertial Measurement Unit on the kite. Luckily, while the kite's orientation is no doubt a useful measurement in any situation, it is not essential for control during crosswind flight, during which the kite is moving rapidly, and the aim is to control the kite's direction of motion rather than its orientation. The velocity angle is the angle the kite's velocity projected onto the plane that is tangent to the sphere at the kite's current position, measured with respect to the sphere's meridian at that point (line of equal azimuthal angle). It has been shown that during crosswind flight, the kite's orientation is approximately the same as its velocity angle, with the advantage of the velocity angle being that it can be inferred from a sequence of position measurements.

The key assumption for deriving the following Cart Model from the Erhard Model is that the kite flies crosswind, which occurs when the lines of the kite do not make a large angle with the wind vector, i.e. when $\frac{\tan \vartheta}{E}<<1$. Importantly, during crosswind flight, the speed of the kite is always greater than the wind speed. We begin by introducing the velocity angle as defined by [16], adapting it to our co-ordinate system. Geometrically, it is defined as the angle the kite's velocity vector, $\dot{\mathbf{p}}$, makes with the following (position dependant) reference vector $\frac{\partial \mathbf{p}}{\partial \vartheta}$. This can be concisely expressed as:

$$
\gamma=\tan ^{-1}\left(\frac{\dot{\varphi} \sin \vartheta}{\dot{\vartheta}}\right)
$$

Developing the expression for the velocity angle (using Equations III.3 and III.4) gives:

$$
\gamma=\tan ^{-1}\left(\frac{-\sin \psi}{\cos \psi-\frac{\tan \vartheta}{E}}\right) .
$$

As $\frac{\tan \vartheta}{E}<<1$ in crosswind flight, it follows that:

$$
\gamma \simeq-\psi .
$$

In other words, the equations above show that the kite flies crosswind in roughly the same direction as it is pointing. This was already noted by [16], [18], where it is called the low sideslip, or drift, assumption. Next, we examine the kite's speed $\|\dot{\mathbf{p}}\|$ when traveling crosswind (we recall that $\mathbf{p}$ is the kite's position in $x, y, z$ coordinates, defined by Equation III.1). The kite's speed relative to the tether length (i.e. in $\left.\mathrm{rad} \cdot \mathrm{s}^{-1}\right)$ is:

$$
\omega_{\mathrm{k}}:=\frac{\|\dot{\mathbf{p}}\|}{r}=\sqrt{(\dot{\varphi} \sin \vartheta)^{2}+(\dot{\vartheta})^{2}} .
$$

Next, inserting the differential Equations III.3 and III.4 gives:

$$
\begin{aligned}
\omega_{\mathrm{k}} & =\sqrt{\left(-\frac{w_{\mathrm{ap}}}{r} \sin \psi\right)^{2}+\left(\frac{w_{\mathrm{ap}}}{r}\left(\cos \psi-\frac{\tan \vartheta}{E}\right)\right)^{2}} \\
& =\frac{w_{\mathrm{ap}}}{r} \sqrt{(\sin \psi)^{2}+\left(\cos \psi-\frac{\tan \vartheta}{E}\right)^{2}} .
\end{aligned}
$$

Finally, as $\frac{\tan \vartheta}{E}<<1$ in crosswind flight:

$$
\omega_{\mathrm{k}} \simeq \frac{w_{\mathrm{ap}}}{r}=\frac{w}{r} E \cos \vartheta .
$$

Hence, during crosswind flight, the kite's speed approximately depends only on the position, and not the orientation, of the kite. Similarly to the above equation, several authors have shown or observed that during crosswind flight, the kite's velocity is approximately equal to the apparent wind negated (e.g., in [16], [18]). Using the definition of $\gamma$ from Equation (III.8) and the expression for the kite's angular velocity from Equation (III.11), the Cart Model for the kite reads:

$$
\begin{aligned}
& \dot{\vartheta}=\omega_{\mathrm{k}} \cos \gamma, \\
& \dot{\varphi}=\frac{\omega_{\mathrm{k}}}{\sin \vartheta} \sin \gamma, \\
& \dot{\gamma}=-\left(\omega_{\mathrm{k}} r g_{\mathrm{s}} \delta+\dot{\varphi} \cos \vartheta\right), \\
& \omega_{\mathrm{k}}=\frac{w}{r} E \cos \vartheta .
\end{aligned}
$$

These dynamics are the same as those of a very simple (nonholonomic) mobile robot (or a 'cart') driving on a sphere, with affine steering dynamics. The control of such a system on a flat surface has already been treated in the robotics literature, see for example [38]. If unlimited steering action is assumed, any path can be achieved, but in reality the steering deflection is bounded. Note that, if $E$ is assumed to be constant (which, as we shall see, is reasonable if the model is to be used to design steering controllers, but not for tether-force optimization), the input required to follow any smooth path can be easily computed, although it may not satisfy the maximum steering-deflection constraint. This compact model is simple enough that the values of the free parameters can easily be identified experimentally, yet it will nonetheless allow good control performance during crosswind flight to be achieved.

\section{Experimental Characterization of the Kite's Turning Be- havior}

At this point, it is important to see how this model compares to actual experimental data. It will be seen that two additional characteristics of the kite's turning behavior must be accounted for. Let the corrected turning rate be defined as:

$$
\dot{\gamma}_{\mathrm{c}}=\dot{\gamma}+\dot{\varphi} \cos \vartheta
$$




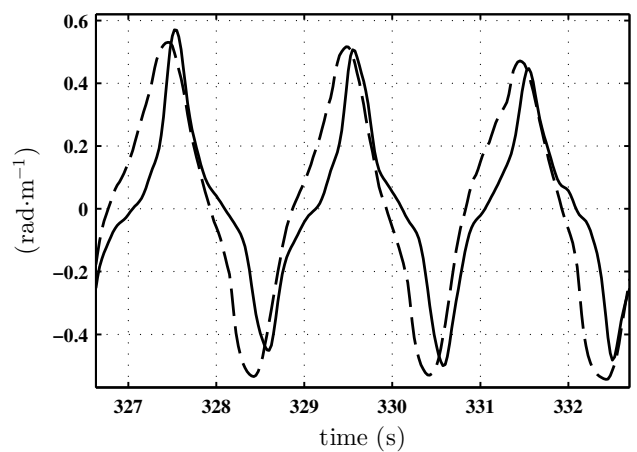

Fig. 4. The scaled steering input, $g_{\mathrm{s}} \delta$ (dashed), with $g_{\mathrm{s}}=1.1 \mathrm{rad} \cdot \mathrm{m}^{-2}$, and $-\frac{\dot{\gamma}_{\mathrm{c}}}{\omega_{\mathrm{k}} r}$ (solid), while the kite flies regular figure-of-eights. Kite: $2.5 \mathrm{~m}^{2}$ Flysurfer Viron, wind speed: $10-15 \mathrm{~m} \cdot \mathrm{s}^{-1}$.

Injecting Equation (III.18) into Equation (III.16) leads to the following proportional relationship:

$$
-\frac{\dot{\gamma}_{\mathrm{c}}}{\omega_{\mathrm{k}} r}=g_{\mathrm{s}} \delta
$$

The constant $g_{\mathrm{s}}$ can be estimated from experimental data, as shown in Figure 4, by finding the value of $g_{\mathrm{s}}$ that results in the two signals overlaying each other. Clearly, $-\frac{\dot{\gamma}_{c}}{\omega_{k} r}$ is only approximately proportional to the steering input $\delta$. More important, there is a significant delay between a change in the steering deflection and the resulting change in the turning rate. Note that this delay occurs at the level of the kite and the lines, as all software and actuation delays inherent in the ground station have already been accounted for in Figure 4. Although not well understood from a modeling perspective, the delay is most likely due to the rotational inertia of the kite and the flexibility of both the lines and the kite itself. This significant delay is a very important aspect of the kite's behavior, and the manner in which it is dealt with will largely affect the control performance.

The second experimentally observable phenomenon is the effect of turning on the line tension. An optimizing control strategy for a kite will generally aim to maximize some function of the line tension, for example the component of the line tension in a particular direction for vehicle traction. The Erhard Model (and hence the Cart Model) predicts that the line tension and the kite's speed simply depend on the angle between the tether and the wind, $\vartheta$, if $E$ is assumed constant. As we shall see, this assumption is not always true, it is certainly a reasonable approximation if the model is used for directional control of the kite, where subtle variations in the kite's speed and the line tension are of secondary importance. However, for optimization, such variations in the line tension are of primal importance. During experiments, it was observed that steering deflections cause a significant reduction in tether tension. This is particularly noticeable for very large steering deflections, which will almost cause the kite to stall, drastically reducing the apparent wind speed, and hence the tether tension. In [30], we proposed to link steering deflections to a reduction in the lift-to-drag ratio using the following empirical law:

$$
E=E_{0}-c \delta^{2},
$$

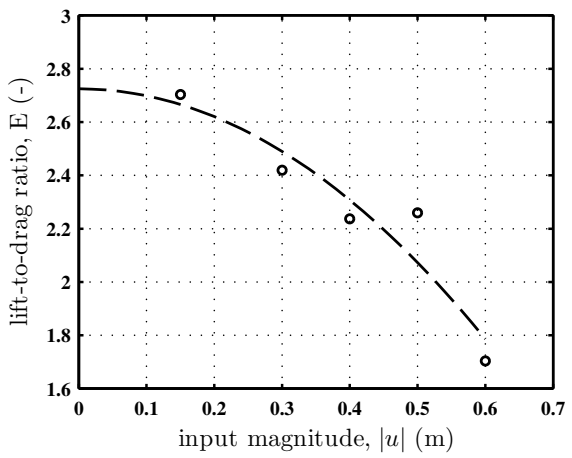

Fig. 5. The lift-to-drag ratio vs. the magnitude of the steering-deflection setpoint, estimated from experimental data using Equation (III.21) (circles), and then fitted to these points according to Equation (III.20) (dashed). Kite: 2.5 $\mathrm{m}^{2}$ Flysurfer Viron, wind speed: $7-10 \mathrm{~m} \cdot \mathrm{s}^{-1}$

where $c$ is a constant that determines how much the kite's lift-to-drag ratio is penalized for a steering deflection. The basic idea is that the two mechanisms by which flexible kites steer, banking and introducing twist along the wing, tend to decrease the kite's effective lift-to-drag ratio, which affects the tether tension. This effect was observed by [39] as part of a very comprehensive aerodynamic simulation study. Both of these effects can be modeled as a decrease in $E$ that depends on the steering deflection $\delta$. A quadratic dependence was chosen, as it is symmetric with respect to $\delta$ (which makes sense, as kites are symmetric, and so should behave identically whether they are steered to the left or to the right) and it fits the experimental data reasonably well, as we will now show. So-called 'bang-bang' experiments were carried out, during which the magnitude of the input was maintained constant, while an operator commuted the sign (steering direction) between positive and negative (left and right) to keep the kite flying crosswind. Based on Equation (III.6), the value of $E$ corresponding to each value of $|\delta|$ was then estimated as:

$$
E(|\delta|)=\frac{1}{t_{\mathrm{f}}-t_{0}} \int_{t_{0}}^{t_{\mathrm{f}}} \frac{w_{\mathrm{ap}}}{\bar{w} \cos \vartheta} d t
$$

where $\bar{w}$ is the average measured wind speed between $t_{0}$ and $t_{\mathrm{f}}$, multiplied by the wind-shear factor of 1.35 . According to the wind-shear model from Equation III.2 (and using a $=0.15$, which corresponds to grassy terrain [3]), it can be expected that the wind speed at $20 \mathrm{~m}$ height is about $35 \%$ greater than that measured at $3 \mathrm{~m}$ height. $w_{\mathrm{ap}}$ is determined according to its definition from the apparent wind vector, and the apparent wind vector is given by $(\bar{w} \hat{x}-\dot{\mathbf{p}})$. A good estimate of $\dot{\mathbf{p}}$ could be obtained based on the differences between successive position measurements. For each value of $|\delta|$, a time period $t_{\mathrm{f}}-t_{0}$ of at least 1 minute (corresponding to more than 30 turns of the kite) was used. The resulting values, along with the fitted curve for $E$, are shown in Figure 5. Figure 6 shows the how the law compares with time-series test data. The instantaneous value of $E$ is estimated as:

$$
E(t)=\frac{w_{\mathrm{ap}}}{w \cos \vartheta},
$$

where $w$ is the measured wind speed, multiplied by the wind shear factor used above. It can be seen that the steering-penalty 


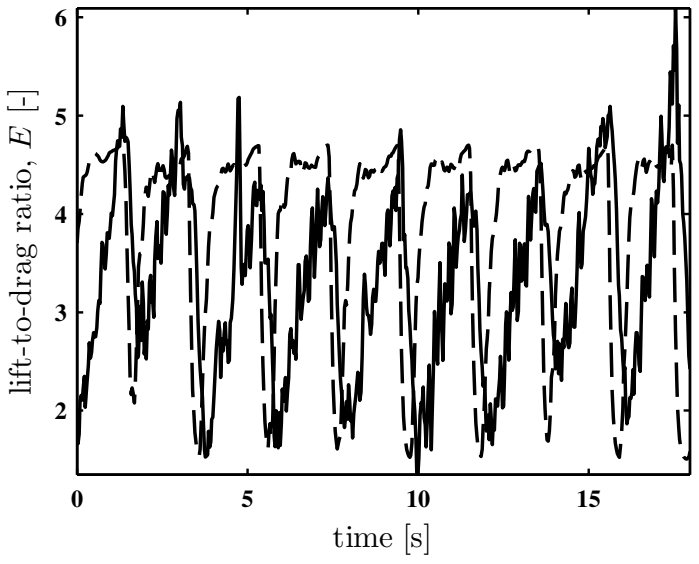

Fig. 6. The instantaneous lift-to-drag ratio for the Apex kite during (autonomous) figure-of-eights. Solid: $E$ estimated from the kite's speed according to Equation (III.22), dashed: $E$ obtained using the steering-penalty law from Equation (III.20) and the parameters in Table I.

TABLE I

AERODYNAMIC MODEL PARAMETERS FOR THE KITES USED.

\begin{tabular}{|c|l|c|c|}
\hline Parameter & Unit & Flysurfer Viron & HQ Apex \\
\hline$A$ & $\mathrm{~m}^{2}$ & 2.5 & 3.5 \\
$g_{\mathrm{s}}$ & $\mathrm{rad} \cdot \mathrm{m}^{-2}$ & 1.1 & 0.9 \\
$c$ & $\mathrm{~m}^{-2}$ & 2.61 & 12.8 \\
$E_{0}$ & - & 2.73 & 4.7 \\
\hline
\end{tabular}

law in Equation (III.20) matches the data reasonably well. There is a notable lag, which we believe can be attributed to the kite's inertia. It can be seen that for both kites large steering deflections result in a reduction of over $30 \%$ in the lift-to-drag ratio, which results in a reduction in line tension of over $50 \%$. Incorporating this steering-penalty law into the model when performing path optimization was key to obtaining meaningful results.

Aerodynamic parameters for the experimental system, estimated during experiments with wind speeds of $7-15 \mathrm{~m} \cdot \mathrm{s}^{-1}$ and 4-5 $\mathrm{m} \cdot \mathrm{s}^{-1}$ for the Viron and Apex kites respectively, are given in Table I. The values of these parameters are only indicative as they were obtained for specific conditions. Although we cannot currently model this precisely, the kite behavior was qualitatively observed to change with wind speed. It is partly for this reason that the control and optimization schemes used in this article and presented next neither rely on a precise model, nor require re-estimation of the model parameters for different wind conditions.

\section{PAth-Following Control}

The control strategy was chosen by comparing a number of different approaches and by building upon the work of other researchers [5], [16], [18]. The novelty is that a) we use position measurements only, b) we use delay compensation, which in our case dramatically improves closed-loop performance, and c) we adapt a path-following approach from the robotics litterature to the case of following variable-curvature paths on a sphere. Using the Cart Model, the path-following cascade control loop shown in Figure 7 can be constructed. Firstly, the missing state, the velocity angle, is estimated from the kite's measured position. This is described in Section IV-A. Next, an "adaptive prediction" block advances the measurements forward in time to counteract the inevitable time delays inherent in the system. These include the delay introduced by the velocity estimation technique, the computational delay, the delay introduced by filters, the actuator response delay and the aforementioned steering delay of the kite of Section III-D. The adaptive prediction block acts in exactly the opposite manner to a time delay by taking the current position measurement and the estimated velocity angle and integrating an adaptive model of the kite forward in time to predict the future values of these states. The adaptive prediction algorithm has been published in a separate article [40] and will not be detailed here. Note that delay is a clear problem for kite control [18], and other compensation approaches have also recently been proposed [37], [41].

The guidance strategy block uses a geometric algorithm to produce a reference velocity angle such that the kite will follow the reference path $\left\{\vartheta_{\mathrm{r}}(\cdot), \varphi_{\mathrm{r}}(\cdot)\right\}$. The guidance strategy is described in Section IV-B. This reference path is itself periodically updated by the RTO algorithm to be described in Section V. A low-level proportional controller tracks the velocity-angle setpoint. Section IV-C shows the impact of delay compensation on this control loop during experiments. Finally, results following different paths are presented in Section IV-D.

\section{A. Velocity-Angle Estimation}

At the sampling instant $n$, an estimate of the kite's velocity vector, $\hat{\mathbf{v}}$, is obtained by simply fitting a velocity vector to a number of previous position estimates $\hat{\mathbf{p}}[n-2 d], \ldots, \hat{\mathbf{p}}[n]$. This is achieved by solving the following least-squares problem:

$$
\{\hat{\mathbf{v}}, \hat{\mathbf{a}}\}=\arg \min _{\{\mathbf{v}, \mathbf{a}\}} \sum_{j=0}^{2 d}\left\|\left(\mathbf{a}-j T_{\mathbf{s}} \mathbf{v}\right)-\hat{\mathbf{p}}[n-j]\right\|^{2},
$$

where $\hat{\mathbf{a}}$, a byproduct of the computation, is an additional (and unused as it will tend to be biased) estimate of the kite's current position (and so is approximately equal to $\hat{\mathbf{p}}$ ). Solving this problem fits a line in $3-\mathrm{D}$ space to the last $2 d+1$ position estimates, and the velocity vector associated with the fit is $\hat{\mathbf{v}}$. This is simpler than using a Kalman Filter, while remaining quite robust. On the other hand, this approach introduces an artificial delay of $d$ sampling periods into the control loop, as it finds the kite's velocity vector $d$ sampling periods in the past.

\section{B. Guidance Algorithm}

This section develops a geometric "vector-field" pathfollowing controller for kites. This type of controller is popular in the unmanned-aerial-vehicle (UAV) community, where it was primarily developed to follow circular and straight-line paths (or composites of these) [42]. Here, it is adapted to follow arbitrary smooth paths (including paths that intersect themselves). Firstly, the controller aims to control the position 


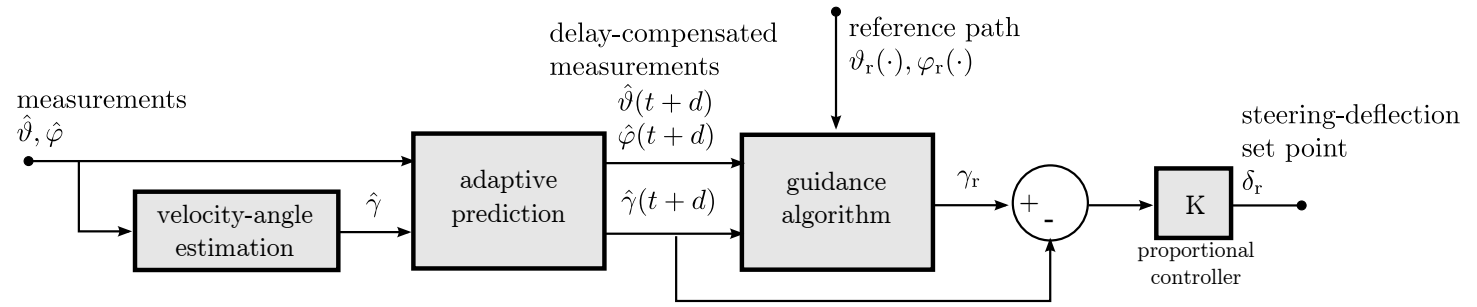

Fig. 7. The path-following cascade control loop.

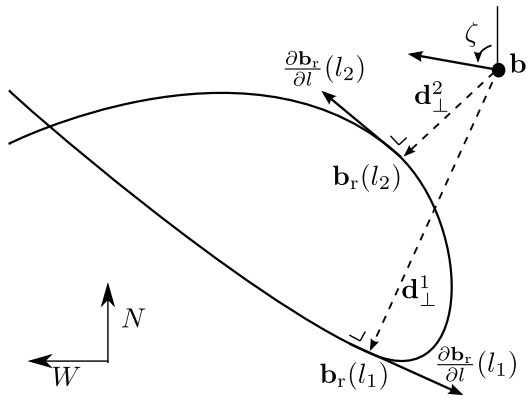

Fig. 8. Path-following controller. Illustration of the kite's position relative to the reference path (all projected onto the $\{N, W\}$ plane shown in Figure $3)$. $\mathbf{b}$ is the kite's position, and $\mathbf{b}_{\mathrm{r}}\left(l_{1}\right)$ and $\mathbf{b}_{\mathrm{r}}\left(l_{2}\right)$ are the two points on the path at which the path's tangent is perpendicular to the line joining the kite position to that point.

of the kite on the $\{N, W\}$ plane, as defined in Section III-B. Recall that this plane is tangent to the sphere upon which the kite can move, at the point $\{\bar{\vartheta}, 0\}$. As the paths of interest are horizontally lying figures-of-eight, this point is chosen as the intersection point of the figure-of-eight reference path, i.e. the reference path's center. The utility of this plane is that it is a local approximation to the sphere that allows the pathfollowing problem to be considered on a flat 2-D surface. The kite's position on the $\{N, W\}$ plane is defined as:

$$
\mathbf{b}=\mathbf{T} \mathbf{p}, \quad \text { with } \quad \mathbf{T}=\left[\begin{array}{ccc}
-\sin \bar{\vartheta} & 0 & \cos \bar{\vartheta} \\
0 & 1 & 0
\end{array}\right] .
$$

The kite's velocity in $\{x, y, z\}$ coordinates is given by:

$$
\dot{\mathbf{p}}=r \mathbf{C}\left[\begin{array}{c}
\dot{\vartheta} \\
\dot{\varphi} \sin \vartheta
\end{array}\right] \text {, with } \mathbf{C}=\left[\begin{array}{cc}
-\sin \vartheta & 0 \\
\cos \vartheta \sin \varphi & \cos \varphi \\
\cos \varphi \cos \vartheta & -\sin \varphi
\end{array}\right]
$$

Next, using Equations (III.14) and (III.15), a relationship between $\gamma$ and the kite's velocity vector projected onto the $\{N, W\}$ plane can be obtained:

$$
\dot{\mathbf{b}}=r \mathbf{T C}\left[\begin{array}{c}
\dot{\vartheta} \\
\dot{\varphi} \sin \vartheta
\end{array}\right] \text {, with }\left[\begin{array}{c}
\dot{\vartheta} \\
\dot{\varphi} \sin \vartheta
\end{array}\right]=\omega_{\mathrm{k}}\left[\begin{array}{c}
\cos \gamma \\
\sin \gamma
\end{array}\right],
$$

where we recall that $\omega_{\mathrm{k}}$ is the kite's speed in $\mathrm{rad} \cdot \mathrm{s}^{-1}$. We define the kite's velocity angle on the $\{N, W\}$ plane as:

$$
\zeta=\angle \dot{\mathbf{b}}=\tan ^{-1}\left(\frac{\dot{\mathbf{b}}_{W}}{\dot{\mathbf{b}}_{N}}\right) \text {. }
$$

The reference path (on the $\{N, W\}$ plane) is denoted $\mathbf{b}_{\mathrm{r}}(l)$, where $l$ is the path length. The points on the path at which the path's tangent is perpendicular to the kite position are $\mathbf{b}_{\mathrm{r}}\left(l_{i}\right)$. The angle of the path at each point is denoted as:

$$
\zeta^{i}=\angle \frac{\partial \mathbf{b}_{\mathrm{r}}}{\partial l}\left(l_{i}\right),
$$

while the vector pointing from the kite to each point is:

$$
\mathbf{d}_{\perp}^{i}=\mathbf{b}_{\mathrm{r}}\left(l_{i}\right)-\mathbf{b} .
$$

A desired velocity angle corresponding to each point is obtained with the classic vector-field law [42]:

$$
\begin{aligned}
\zeta_{\mathrm{d}}^{i}= & \zeta^{i}+\zeta_{\mathrm{e}}\left(\frac{\left\|\mathbf{d}_{\perp}^{i}\right\|}{d_{\max }}\right)^{\beta} \times \operatorname{sgn}\left(\angle\left(\mathbf{d}_{\perp}^{i}-\frac{\partial \mathbf{b}_{\mathrm{r}}}{\partial l}\left(l_{i}\right)\right)\right) \\
& +\alpha \frac{\partial^{2} \mathbf{b}_{\mathrm{r}}}{\partial l^{2}}\left(l_{i}\right),
\end{aligned}
$$

where the entry velocity angle $\zeta_{\mathrm{e}}$ and the coefficient $\beta>1$ are tuning parameters. The final term is a new (at least in the context of the vector-field controller) curvature-compensation term that helps the controller follow a curved path. The curvature of the path indicates the rate of change of the path's angle (direction). Thanks to the curvature compensation (which can be varied by adjusting $\alpha$ ), the controller anticipates curves in the path. Finally, the reference velocity angle is selected as the $\zeta_{\mathrm{d}}^{i}$ that is closest to the kite's current velocity angle:

$$
\zeta_{\mathrm{r}}=\zeta_{\mathrm{d}}^{i_{\mathrm{r}}}, \quad i_{\mathrm{r}}=\arg \min _{i} \quad\left|\zeta-\zeta_{\mathrm{d}}^{i}\right| .
$$

The reference velocity angle in $\{N, W\}$ coordinates can be translated back into a reference signal for the velocity-angle control loop by inverting Equation IV.4:

$$
\gamma_{\mathrm{r}}=\tan ^{-1}\left(\frac{x_{2}}{x_{1}}\right), \text { with } \mathbf{x}=(\mathbf{T C})^{-1}\left[\begin{array}{l}
\cos \zeta_{\mathrm{r}} \\
\sin \zeta_{\mathrm{r}}
\end{array}\right] .
$$

\section{Velocity-Angle Control}

We will show next that for the velocity angle, a simple proportional controller yields good performance, provided delay is compensated for. Firstly, note that the final term in Equation (III.16) is generally relatively small, meaning that the velocity-angle dynamics are approximately those of an integrator. Hence, a proportional controller can be expected to yield good performance. Moreover, a fixed gain is sufficient as, according to this equation, the responsiveness to the control input is proportional to the kite's speed. The performance during an experiment with adaptive prediction is shown in Figure 9. The small assymetry in the tracking is probably due to a slight misalignment of the reference trajectory with the 


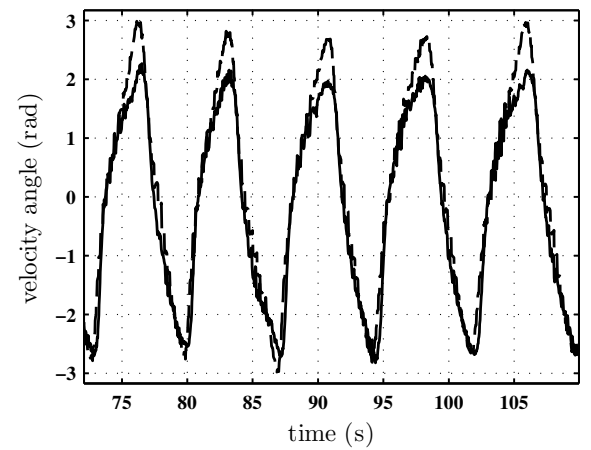

Fig. 9. Performance of the velocity-angle control loop during autonomous figure-of-eights with adaptive prediction: the reference signal $\gamma_{\mathrm{r}}(t)$ (dashed), and the controlled variable $\hat{\gamma}(t+d)$ (solid).

wind vector. The considerably worse performance obtained during an experiment without adaptive prediction is shown in Fig. 10. The oscillations around the reference signal are typical of proportional control applied to a first-order system with significant time delay. In our case, there is a total delay of about $260 \mathrm{~ms}$, during which the kite can travel up to 10 $\mathrm{m}$, with a horizontal width of the entire reference path being potentially limited to $15 \mathrm{~m}$. A very important peculiarity of path-following control can be observed, namely, oscillations of the controlled variable cause oscillations of the reference signal. This is because the reference signal depends on the kite's position. These variations in the reference signal are likely to cause further oscillations in the controlled variable, as the poorly performing controller tries to track them. The overall effect is poor path following and, for this reason, it was found that the response of the velocity control loop must absolutely not overshoot or oscillate. Hence, it was best to choose a conservative value (i.e. a low one) for the proportional gain. A corollary is that it is not sufficient to study the stability properties of the velocity-angle control loop alone. Even if the velocity-angle control loop is theoretically stable, time variation of the reference signal, which the velocity-angle control loop itself may cause, can still result in oscillations of increasing amplitude around the reference path. In this work, no attempt was made to analyze the theoretical stability properties of the entire path-following controller.

\section{Results}

The tracking of different reference paths is shown in Figures 11, 12 and 13. Each of these graphs represents 10 minutes of experimental data, during which the kite flies roughly 150 loops. It can be seen that the controller is very consistent, that is, the path followed by the kite is very similar from one loop to the next. The path followed by the kite is not exactly the reference path, and there is a small but consistent offset between the two. This is because the vector-field control law is heuristic. It is essentially a path-following equivalent of a proportional-derivative (PD) controller. Hence, while it can be tuned to provide good and robust performance, it will not provide exact path-following.

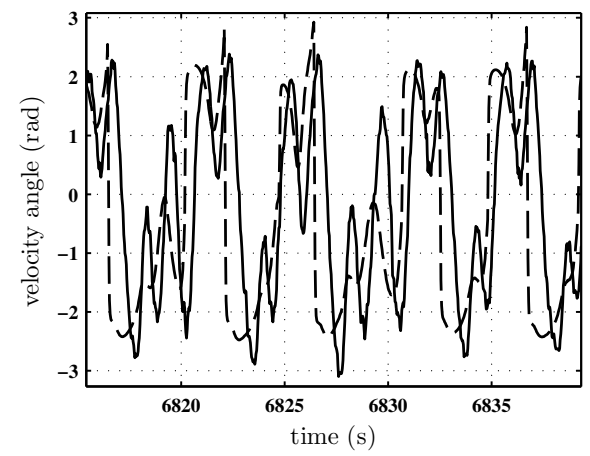

Fig. 10. Performance of the velocity-angle control loop during autonomous figure-of-eights without adaptive prediction: the reference signal $\gamma_{\mathrm{r}}(t)$ (dashed), and the controlled variable $\hat{\gamma}(t)$ (solid). Kite: $2.5 \mathrm{~m}^{2}$ Flysurfer Viron, wind speed: $6-8 \mathrm{~m} \cdot \mathrm{s}^{-1}$

The primary weakness of the controller is very light winds. In very light winds (less than $2 \mathrm{~m} \cdot \mathrm{s}^{-1}$ ), the kites becomes prone to stalling during turns. This can be explained by the reduction in lift-to-drag ratio caused by a steering deflection (Equation III.20). Once the kite slows considerably, the assumption of crosswind flight no longer holds. The consequence is that the proportional relationship linking the change in velocity angle to the steering deflection no longer holds (the behavior of the kite becomes very complex during a stall), so the velocity angle control loop ceases to function correctly, and control of the kite's movement is lost. If the kite picks up enough speed again before coming too close to the ground, the algorithm may recover control, otherwise the kite will crash. An experienced human operator using manual controls is far superior in these winds, as they will adjust the kite's angle of attack to avoid such stalls.

Heavy winds, in theory, present no apparent issues for this type of controller, assuming material failures do not occur due to the higher line forces and the sampling rate is fast enough to handle the faster dynamics. This was our observation when testing the ancestors of this algorithm in strong winds, when we were able to achieve autonomous flight in winds of up to $15 \mathrm{~m} \cdot \mathrm{s}^{-1}$ with algorithms we believe were inferior to the one presented in this article. However, the exact algorithm presented in this paper was tested in light to moderate winds.

\section{Optimization of THE Kite's EfFiciency}

The previous section was about ensuring that the kite's autonomous flight is predictable and robust to wind variations. It is also desirable that the kite should fly as efficiently as possible, which is the focus of this section. In this work the average line tension, that is how much the kite "pulls", is used as a measure of efficiency. This is closely related to the efficiency measures for electricity-generating kites or for ship-towing kites. In theory, once a model of the kite and the wind is available, numerical optimization can be used to compute the optimal reference path that yields maximal efficiency. However, this approach is difficult in practice, as the models at our disposal are relatively inaccurate. In addition, due to changing wind conditions, the optimal reference path 


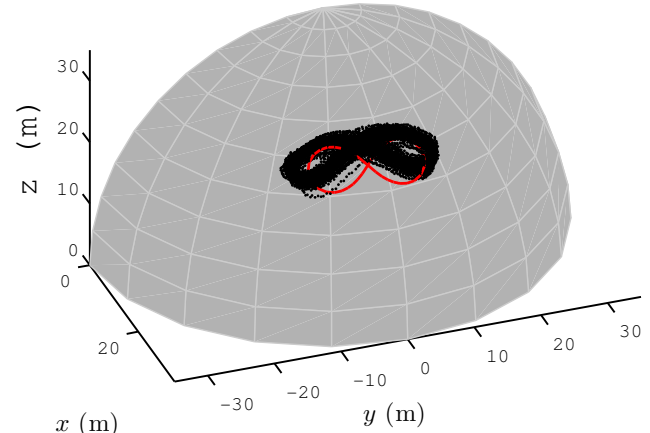

Fig. 11. The kite's position (dots) during 10 minutes of autonomous flight, tracking the high, narrow reference path shown in red. The kite is restrained to flying on the gray quarter sphere.

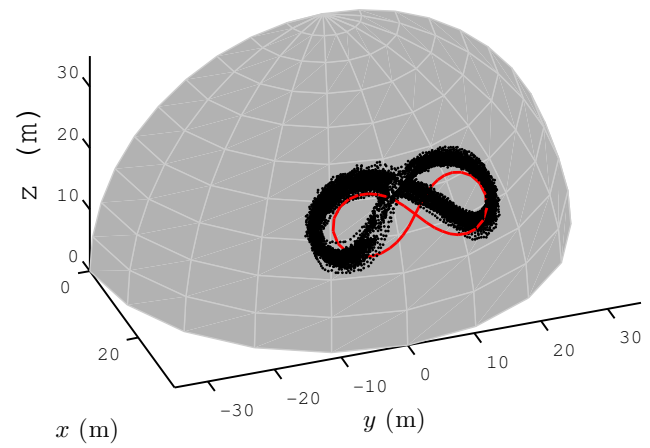

Fig. 12. The kite's position (dots) during 10 minutes of autonomous flight, tracking the low, narrow reference path shown in red.

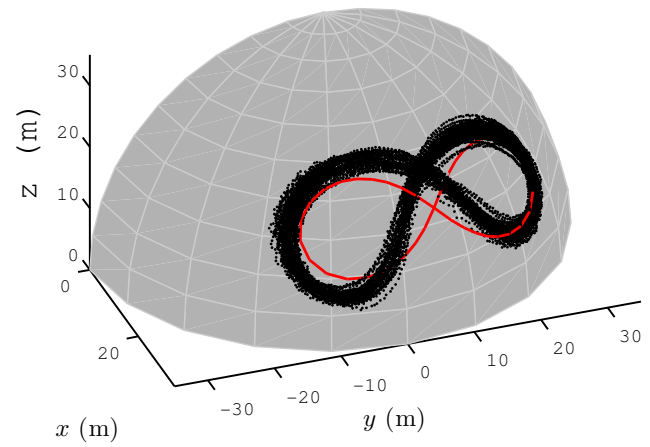

Fig. 13. The kite's position (dots) during 10 minutes of autonomous flight, tracking the low, wide reference path shown in red.

may vary over time. The solution adopted here is to implement a real-time optimization (RTO) layer that adjusts the reference path in order to maximize efficiency. The RTO algorithm uses a combination of numerical optimization and recently obtained measurements. This feedback component ensures robustness to modeling errors, control errors and disturbances. The reference path is parametrized with a finite number of parameters, which are the decision variables of the RTO layer.

\section{A. RTO using Modifier Adaptation (MA)}

A RTO method called Modifier Adaptation (MA) is used. This method was initially developed to address the negative effect of modeling inaccuracies on the results of numerical optimization for multivariable industrial processes [43]. With MA the decision variables (RTO inputs), $\mathbf{u}$ are iteratively adjusted in order to minimize a cost (or maximize a profit function), $\phi_{\mathrm{p}}$, while respecting operational constraints: $\mathrm{g}_{\mathrm{p}} \leq$ 0. Although $\phi_{\mathrm{p}}$ and $\mathbf{g}_{\mathrm{p}}$ are quantities that are measurable, which the user defines as functions of measured outputs of the system, the functions mapping $\mathbf{u}$ to $\phi_{\mathrm{p}}$ and $\mathbf{g}_{\mathrm{p}}$ are generally not perfectly known (this would require perfect knowledge of the system). Instead, an imperfect model can be used to define model-based cost and constraint functions: $\phi(\mathbf{u}, \boldsymbol{\theta})$ and $\mathbf{g}(\mathbf{u}, \boldsymbol{\theta})$, which depends on model parameters $\boldsymbol{\theta}$. This gives a model-based optimization problem, which is essentially used as a starting point for MA:

$$
\begin{aligned}
& \mathbf{u}^{*}(\boldsymbol{\theta})=\underset{\mathbf{u}}{\operatorname{argmin}} \phi(\mathbf{u}, \boldsymbol{\theta}) \\
& \text { subject to } \mathbf{g}(\mathbf{u}, \boldsymbol{\theta}) \leq \mathbf{0} .
\end{aligned}
$$

Solving this model-based optimization problem with the best available estimated (nominal) values of the parameters, $\boldsymbol{\theta}_{0}$, yields $\mathbf{u}^{*}\left(\boldsymbol{\theta}_{0}\right)$, which usually will not be optimal for the real system. Thus, MA proposes to modify the aforementioned model-based optimization at each iteration by adding measurement-based modifications to $\phi(\mathbf{u}, \boldsymbol{\theta})$ and $\mathbf{g}(\mathbf{u}, \boldsymbol{\theta})$. The main advantage of MA lies in its ability to converge to the true plant optimum, despite parametric uncertainty and disturbances (e.g. wrong values of $\boldsymbol{\theta}$ ) and structural plantmodel mismatch (i.e. structural differences between $\phi$ and $\phi_{\mathrm{p}}$ and/or $\mathbf{g}$ and $\mathbf{g}_{\mathrm{p}}$ ), provided some fairly relaxed adequacy conditions are met. This is made possible by the fact that the modifications of $\phi(\mathbf{u}, \boldsymbol{\theta})$ and $\mathbf{g}(\mathbf{u}, \boldsymbol{\theta})$ are affine-in-input, with the corresponding slopes being the differences between the estimated (from measured data) and modeled cost and constraint gradients. Full details regarding the theoretical properties, the initialization and the tuning of the specific algorithm used for the experimental optimization in this article can be found in [29], [44]. Estimating the gradients of the plant cost and constraint functions typically requires a certain amount of 'exploration' (zig-zagging around in the RTO input space), and this effort increases with the number of RTO inputs. For this reason, it is desirable to chose as few RTO inputs as possible in order to favor more rapid convergence. This is particularly relevant when optimizing a continuous path (which is our case), as arbitrarily many decision variables could be chosen.

\section{B. MA for Kite Path Planning}

In this work, the aim is to maximize the average line tension, and so the measured cost is defined as:

$$
\phi_{\mathrm{p}}(\mathbf{u}):=-\bar{T},
$$

where $\bar{T}$ is the average line tension measured during $N_{\text {avg }}$ cycles following the (periodic) reference path. The only operational constraint is that the kite must fly above a certain 
minimum altitude, $z_{\min }$, and so the measured plant constraint is defined as:

$$
\mathrm{g}_{\mathrm{p}}:=z_{\min }-z_{\mathrm{L}}
$$

where $z_{\mathrm{L}}$ is the lowest altitude attained by the kite during $N_{\text {avg }}$ cycles. The decision variables $\mathbf{u}$ must also be defined in terms of both nature and number. This is a critical choice since too many degrees of freedom will increase the exploration load, while the primary objective is to optimize the performance. In this work, the inputs are chosen as the parameters that determine the height and the curvature of the reference path. The reference path is parametrized by $\mathbf{u}=\left[\begin{array}{ll}u_{1} & u_{2}\end{array}\right]$ in the following manner:

$\left[\begin{array}{l}\vartheta_{\mathrm{r}}(l) \\ \varphi_{\mathrm{r}}(l)\end{array}\right]=\left[\begin{array}{c}\vartheta_{\mathrm{r}}^{*}\left(l, \boldsymbol{\theta}_{0}\right) \\ \varphi_{\mathrm{r}}^{*}\left(l, \boldsymbol{\theta}_{0}\right)\end{array}\right]+u_{1}\left[\begin{array}{c}\Delta \vartheta_{\mathrm{H}}(l) \\ \Delta \varphi_{\mathrm{H}}(l)\end{array}\right]+u_{2}\left[\begin{array}{c}\Delta \vartheta_{\mathrm{C}}(l) \\ \Delta \varphi_{\mathrm{C}}(l)\end{array}\right]$,

where $\left\{\vartheta_{\mathrm{r}}^{*}(l), \varphi_{\mathrm{r}}^{*}(l)\right\}$ is the nominal optimal reference path calculated using the model with the parameters from Table I. $\Delta \vartheta_{\mathrm{H}}(l), \Delta \varphi_{\mathrm{H}}(l)$ and $\Delta \vartheta_{\mathrm{C}}(l), \Delta \varphi_{\mathrm{C}}(l)$ are variations that cause raising and widening of the reference path, respectively. The influence of these variations is shown in Figures 14 and 15. This particular choice of $\mathbf{u}$ is based on a mathematical sensitivity analysis with respect to the model uncertainty, the full details of which are given in [29]. Note that allowing the RTO layer to adjust the reference path in this way allows the generation of many different "figures-of-eight" and, from the practical point of view, can be justified as follows:

- The height of the trajectory can be adjusted to suit the wind shear.

- The curvature (or the width) of the trajectory can be adapted to suit the kite's turning behavior.

Finally, the model-based cost function $\phi(\mathbf{u})$ is deduced from the Cart Model developed in Section III-C. This function returns the line tension that is theoretically obtained if the reference path defined by $\mathbf{u}$ is exactly followed. The RTO block diagram is given in Figure 16.

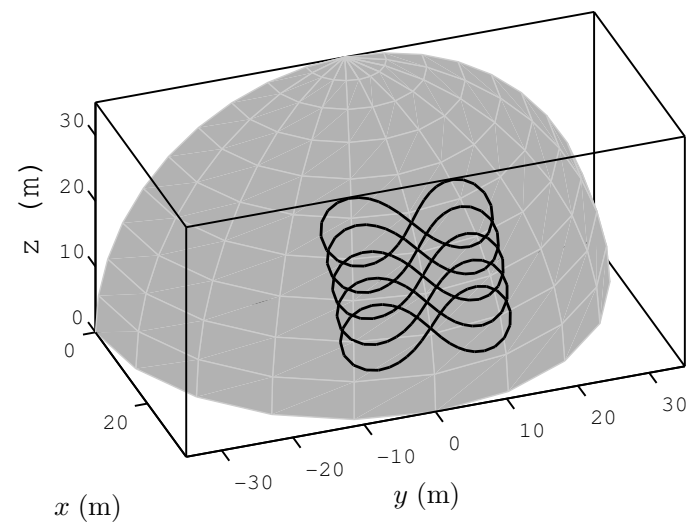

Fig. 14. Reference path for different values of $u_{1}=$ $\{-0.2,-0.1,0,0.1,0.2\}$, with $u_{2}=0$.

\section{Reducing the Effect of Noise}

It is important to characterize the noise affecting the measured cost and constraints. The estimation of experimental

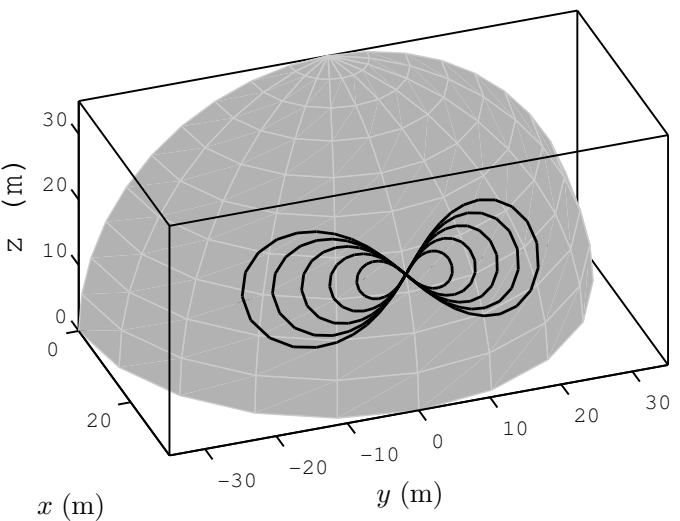

Fig. 15. Reference path for different values of $u_{2}=$ $\{-0.2,-0.1,0,0.1,0.2\}$, with $u_{1}=0$.

gradients can be very badly affected by high-frequency noise, which in turn can lead to unreasonable optimized inputs. The average line tension and the minimum altitude per path cycle are shown in Figure 17, while the kite followed the same reference path for 6 minutes. The minimum height is relatively consistent, with variations of mostly $\pm 1 \mathrm{~m}$, illustrating the consistency of the path-following controller. However, the average line tension is extremely variable, ranging from 60 $\mathrm{kg}$ to $120 \mathrm{~kg}$, that is, with up to $35 \%$ noise. This would be considered extreme noise in the RTO literature. This noise is not induced by variable controller performance, as the kite follows almost exactly the same path during each cycle. Rather, the noise is caused by wind variations. The wind speed measured at the ground station during the same experiment is shown in Figure 18. The measured wind speed varies significantly over time, and there is a rough correlation between the variations in the measured wind speed and the line tension. High-frequency variations in the wind direction also affect the line tension. The path is centered with respect to the average wind direction (the $\mathrm{x}$-axis in Figure 3 is continually realigned with the average measured wind direction over the past 15 minutes). However, the measured wind direction can easily veer by up to 15 degrees for shorter periods. In theory, the measured wind speed and direction could probably be used to scale the average measured line tension in order to reduce the 'noise'. However, in this work, a simpler approach was adopted.

The simple solution to dealing with noise is to partially remove it via averaging. The effect of averaging the line tension over $N_{\text {avg }}=7$ path cycles is apparent in Figure 19. This reduces the noise to a manageable (if still quite high) level. The expense of this is that the RTO algorithm only iterates every 7 path cycles, and thus proceeds more slowly.

\section{RTO Results}

The RTO algorithm's performance was tested over several days of experiments. Depending on the conditions, the attained optimal reference path was quite different. For example, in light winds, the path tended to be much wider (large values of $u_{2}$ ) than in stronger winds. This makes practical sense as the 


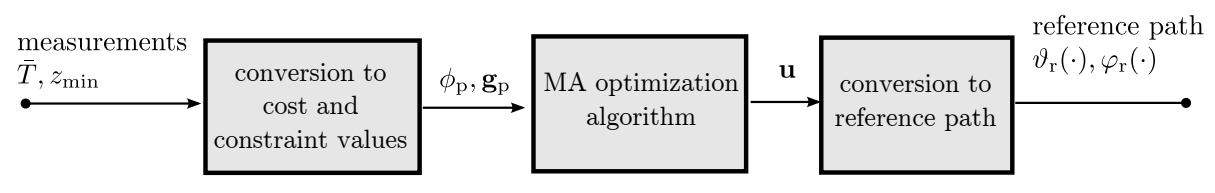

Fig. 16. RTO block diagram.
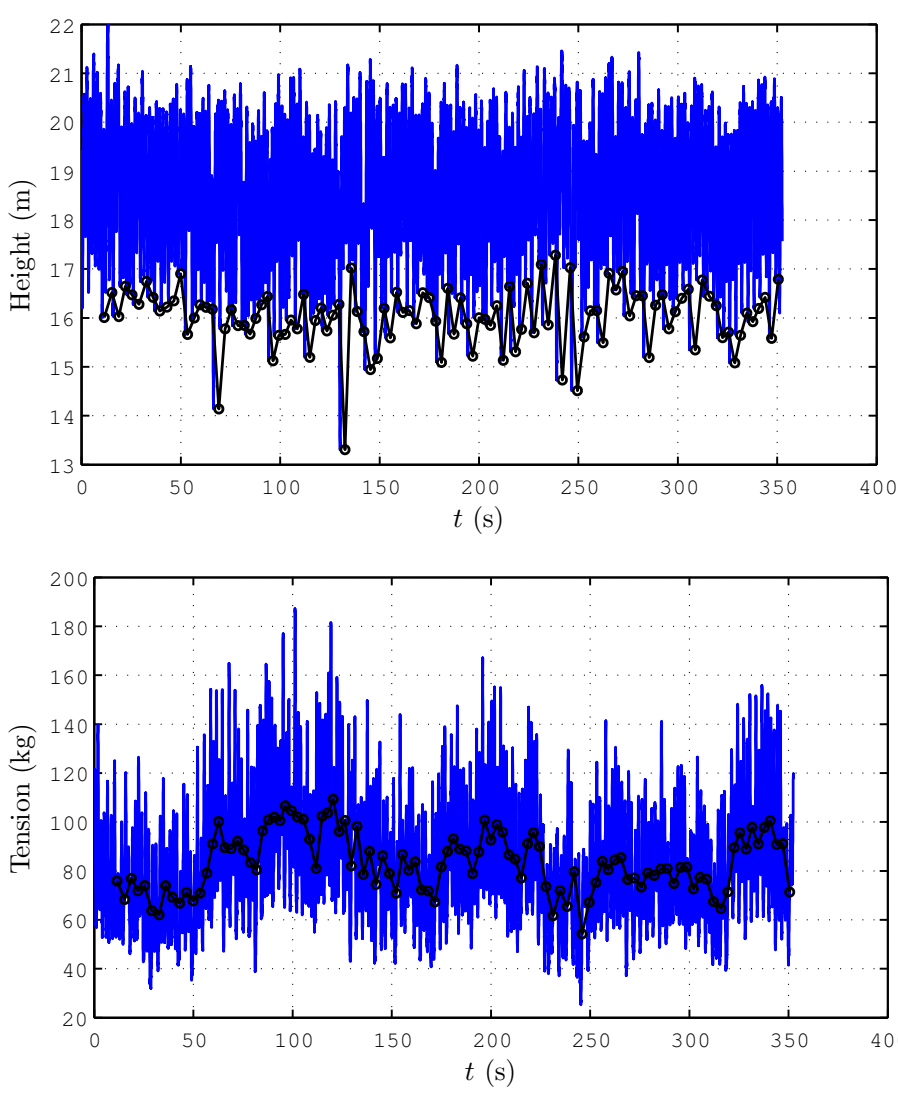

Fig. 17. The kite's altitude and the measured line tension during 6 minutes following a constant reference path (blue). The minimum attained altitude and the average line tension per path cycle (black).

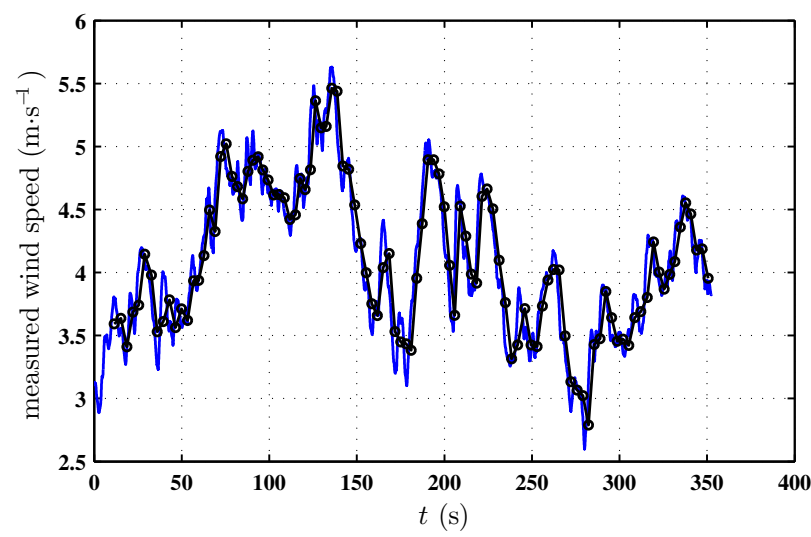

Fig. 18. The wind speed measured at the ground station during the experiment shown in Figure 17 (blue), and the average wind speed per reference-path cycle (black dots).

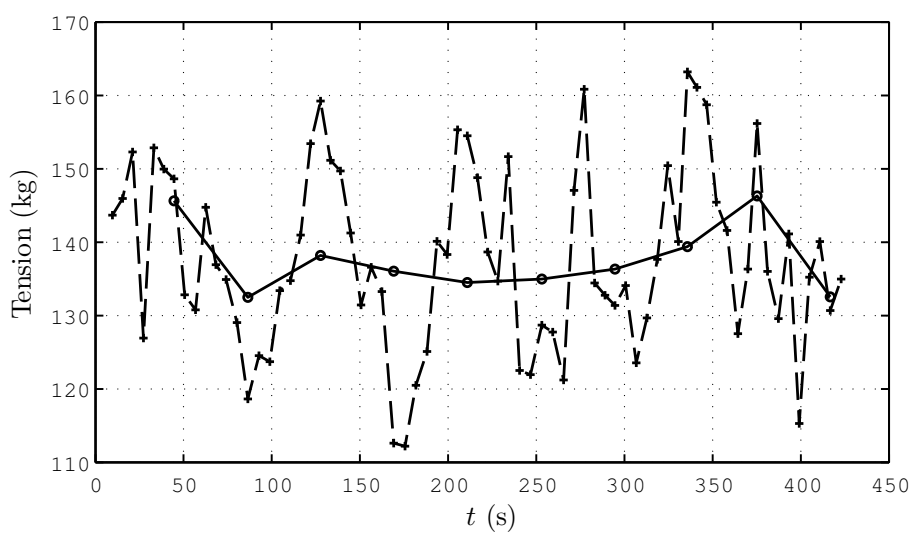

Fig. 19. No averaging (dashed with crosses), averaging over $N_{\mathrm{avg}}=7$ path cycles (solid with circles). During this experiment, the kite followed a constant reference path.

kite becomes far more sluggish and unresponsive to steering at low wind speeds; hence, only gently curving paths can be followed efficiently. However, in order to verify that the RTO algorithm performs correctly, it is useful to test it many times under near-identical conditions, namely, with constant wind speed and constant controller parameters. Unfortunately, meeting the first condition is complicated by the ever-changing wind conditions, Nonetheless, at one point, several hours of continuous experiments were carried out in relatively constant wind conditions without any adjustments being made to the experimental setup, and these results are presented next.

Figure 20 shows the line tension and the kite's altitude during 30 minutes of autonomous flight. During the first 7 minutes (13 iterations) and the last 5 minutes (10 iterations), the kite follows a constant reference path that is rather high and narrow, resulting in a low average line tension, about 80 $\mathrm{kg}$. The RTO algorithm markedly improved the average line tension, increasing it to about $135 \mathrm{~kg}$, during the intermediate 22 minutes that are depicted with a grey-shaded area on Figure 20. The decision variables during this experiment are shown in Figure 21. The zig-zagging behavior is in part caused by the MA algorithm exciting the process in order to estimate experimental gradients (exploration), and in part due to the effect of wind-induced noise. The RTO algorithm is constrained to taking small steps, which introduces a certain robustness to noise. Although it zig-zags, it on average it moves towards the plant optimum.

Next, in order to verify that the RTO algorithm does indeed converge to a neighborhood of the plant optimum, an experimental study was carried out to see how the average line tension varies with respect to the path followed by the kite. This consisted of measuring the average line tension over 10 

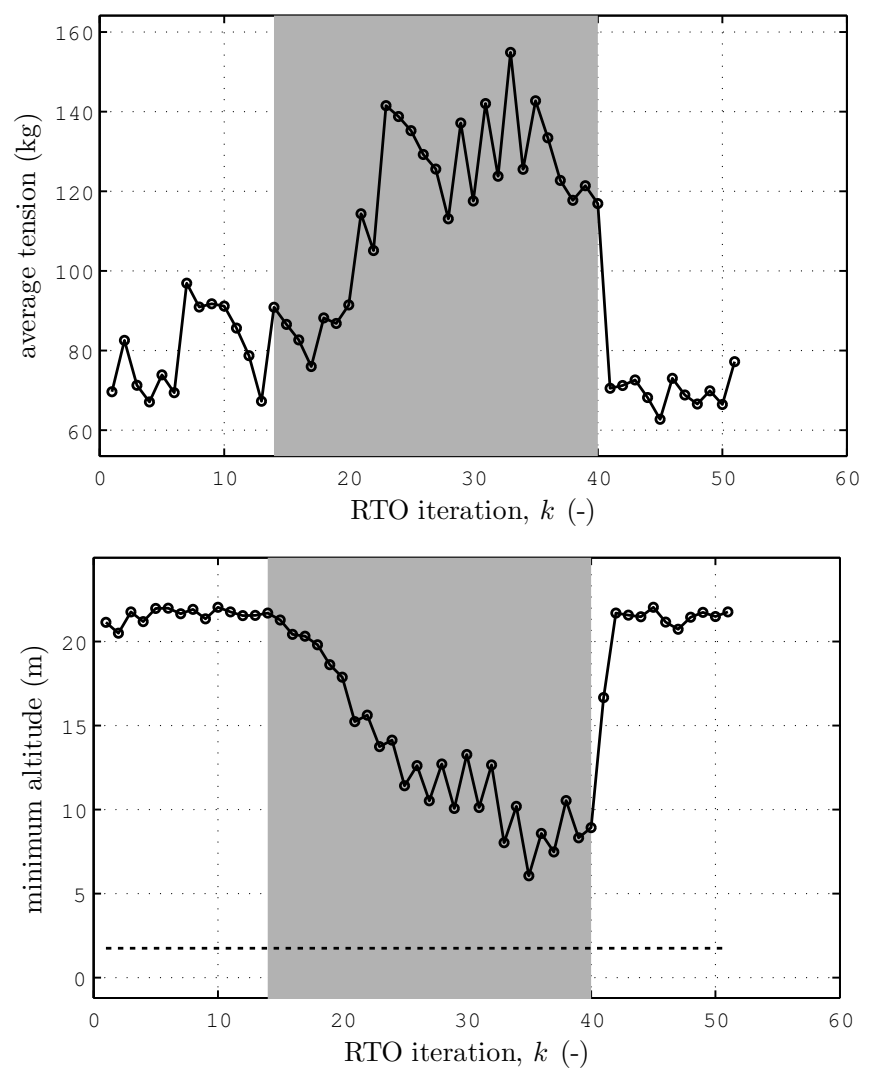

Fig. 20. Performance of the MA algorithm with $N_{\text {avg }}=7$. Each circle is the average/minimum value for the tension/altitude during $N_{\text {avg }}$ path cycles. The dotted line indicates the minimum height constraint. The RTO algorithm was activated during the shaded iterations. The total experiment lasted 29 minutes, and the RTO algorithm was active for 17 minutes.

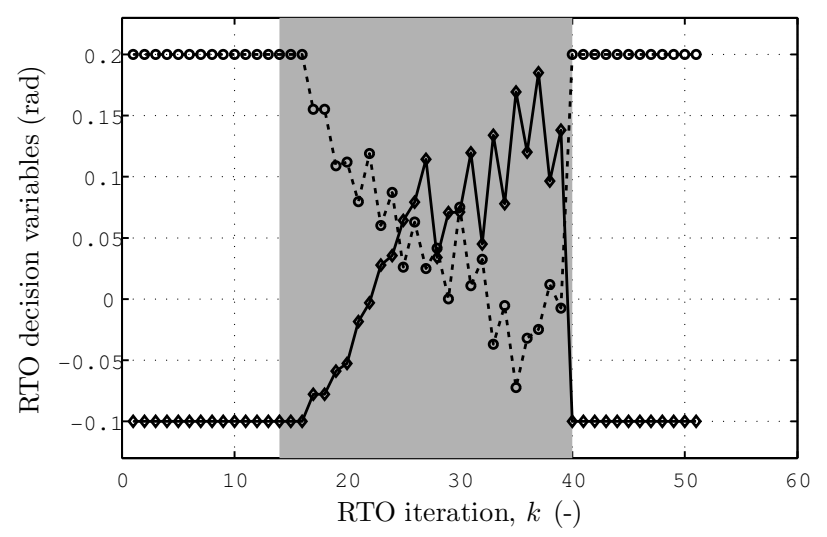

Fig. 21. The decision variables, $u_{1}$ (dashed), and $u_{2}$ (solid) during the experiment shown in Figure 20. minutes while repetitively following a single reference path. This was repeated for 9 different paths. Next, the average line tension that would be obtained for paths between those that were measured was inferred by interpolating between the measured average tensions using a piecewise-cubic interpolation algorithm. The resulting surface is shown in Figure 22. Note that the average measured wind speed was relatively constant during the entire experimental study. Also, it was carried out immediately after the experiment shown in Figure 20, and the conditions were essentially the same. It can be observed that the maximum attainable average line tension is about $130-140 \mathrm{~kg}$. It is interesting to note that the nominal optimal solution that was calculated using the model, corresponding to $u_{1}=u_{2}=0$, results in an average line tension of about 115 $\mathrm{kg}$. Hence, relying only on model-based optimization would have resulted in an optimality loss of about $15-20 \%$ in this case. Figure 23 superimposes the MA algorithm's path on this contour. It is readily seen that, despite the zig-zagging behavior, the algorithm converges to the neighborhood of the plant optimum.

A note on the line-tension magnitude: some readers may be surprised that a $3.5 \mathrm{~m}^{2}$ kite can produce such large line tension in a gentle breeze, however the experimental values displayed here do actually agree with the theory. Firstly, the HQ Apex kite is a high-performance Ram-air kite. As is typical of Ramair kites, the bridle keeps the wing quite flat, and so for its area it produces more line tension than a typical kite-surfing LEI (Leading Edge Inflatible) kite, which are used in some of the existing experimental studies. From experimental flight data, we estimate the HQ Apex has a lift-to-drag ratio ( $E_{0}$ value) of about 4.7. Secondly, the wind speed measurements in this article are for a height of $3 \mathrm{~m}$ above the ground, not at the kite's height. According to the wind-shear model from Equation III.2 (and using a $=0.15$, which corresponds to grassy terrain [3]), it can be expected that the wind speed at $20 \mathrm{~m}$ above the ground is about $35 \%$ greater. Finally, given the air temperature of about $0{ }^{\circ} \mathrm{C}$ during the tests for which linetension is shown, the air density was relatively high, probably about $1.3 \mathrm{~kg} \cdot \mathrm{m}^{3}$. Inserting these values into Equation III.7, with a measured wind speed of $4.5 \mathrm{~m} \cdot \mathrm{s}^{-1}$, when the kite is 20 $\mathrm{m}$ above the ground, directly downwind, and with no steering deflection, we can expect a line tension of 1332 Newtons.

\section{Discussion AND CONCLUSIONS}

The two-layer control and optimization algorithm for kites described in this paper was designed for, and implemented on, a particular experimental setup. However, we believe that a number of useful conclusions can be drawn regarding the control and optimization of kite-power systems in general.

Firstly, while additional measurements are certainly useful, good path-following control (of a kite flying roughly crosswind) can be achieved using only measurements of the kite's position. However, delay must be either eliminated or compensated for as, due to the kite's speed, even a small delay can cause oscillations around the reference path.

Secondly, the average line tension varies significantly depending on the path followed by the kite, to the extent that 


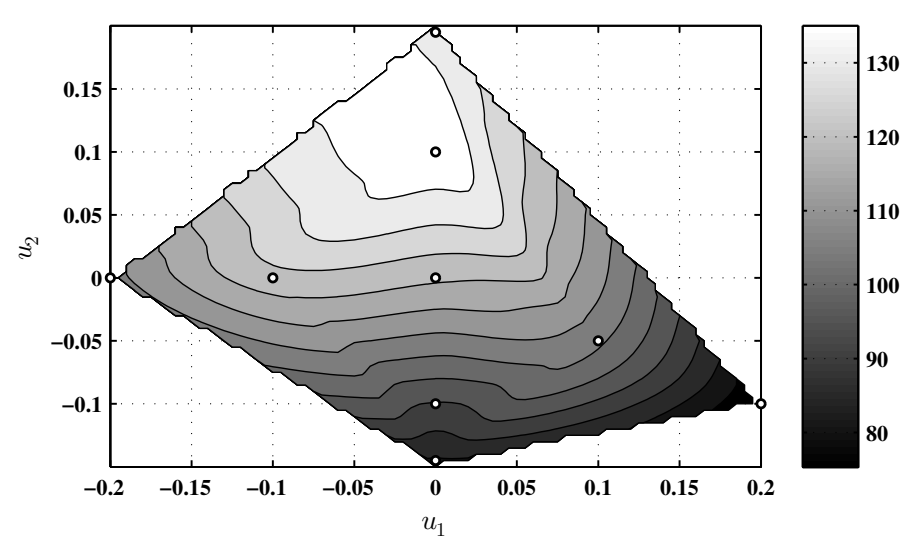

Fig. 22. Contour plot of the average line tension in $\mathrm{kg}$ (shading) per figure-ofeight vs. u. At each of the data points (circles), the average line tension during 10 minutes of experimental data was recorded. The surface was estimated by performing a piecewise-cubic interpolation of these data points.

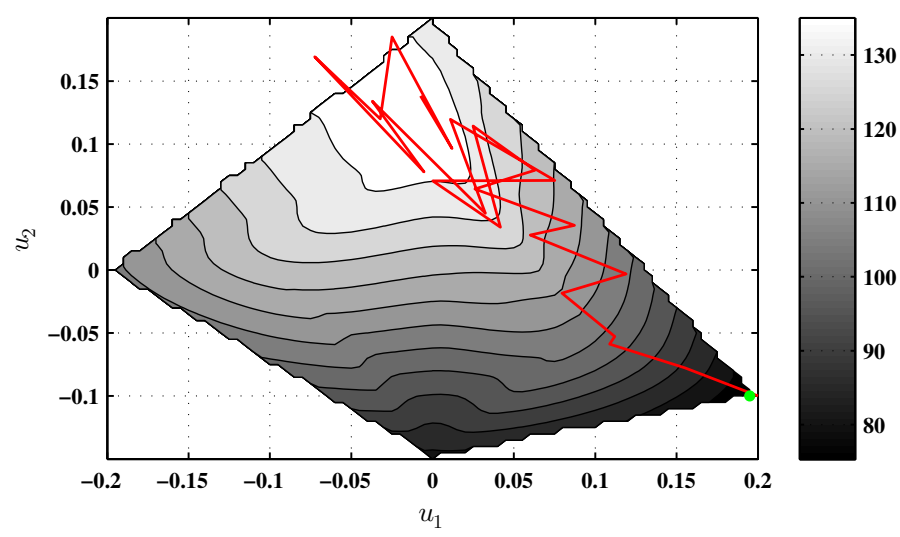

Fig. 23. Contour plot of the attainable average line tension, as shown in Figure 22. The path taken by the MA algorithm is in red, with the green dot being the initial point.

serious attention should be given to the kite's path if efficiency is to be maximized. It is questionable whether model-based optimization alone can calculate an efficient path. Indeed, in this study, using the model's optimal path would have resulted in a $15-20 \%$ lower average line tension compared to the plant optimal path, despite calibrating the model (albeit at quite different wind speeds) using experimental data. It is true that online parameter estimation might reduce this optimality gap somewhat, however designing an estimator that can effectively cope with unmodeled behavior and the nonlinear dynamic model is not at all trivial. It is also true that a relatively simplistic model was used, yet more complex models, although they would certainly lead to a smaller loss in average line tension, may not be practical for two main reasons: (i) they are often too computationally demanding for path optimization, and (ii) they cannot easily account for changes in wind conditions. The solution presented here is to use RTO to constantly update the reference path in order to continuously react to wind and system variations.

How do the algorithms described here apply to the main commercial situations? Currently, there exist many different systems, and the methods described in this paper are more 30 suited to some than to others.

- Modeling: In terms of dynamic modeling, the approach in this paper is suitable for flexible power kites. It does not assume any particular kite geometry or size, and it is equally applicable to a $3-\mathrm{m}^{2}$ or $300-\mathrm{m}^{2}$ kite. As it is a very intuitive and simple model, it can easily be modified to take into account effects such as nonlinear steering dynamics, a variable angle-of-attack or a varying tether length. More accurate detailed models of flexible kites have yet to reach maturity and in the meantime simple tendency models such as this should be preferred. Rigid kites, on the other hand, can benefit from the much more advanced modeling techniques available from classical flight dynamics.

- Control: The path-following controller is, in theory, applicable to any power kite flying in crosswind mode, with some modification. Our approach of estimating the kite's position from line angles is only accurate for short lines. Tethers in excess of $100 \mathrm{~m}$ curve in an unpredictable manner, under the effect of gravity, the tether inertia, and aerodynamic drag. In this case, the kites position and velocity must be estimated using other methods (typically using a strapdown Inertial Measurement Unit and Global Navigation Satellite System). Dead time (delay in the feedback loop) may increase for a larger system, due to tether sag and communication times. This may be a problem, as the dynamics of the velocity angle remain the same. Our controller, using the delay compensation algorithm, could handle a delay of $250 \mathrm{~ms}$ in the feedback loop, but it could probably not tolerate much more. Luckily with a larger setup, the distances involved are much larger, and it will usually be acceptable to stray further from the reference path. Therefore, the controller can be tuned such that it is less aggressive and will tolerate more delay. Nonetheless, for very long tether lengths, it is probable that the kite must be actuated by an airborne actuation system to avoid a prohibitive delay. In any case, the low-level velocity-angle controller should be designed and tuned on a system-to-system basis, as different kites can have different steering behavior and mechanisms. The geometric guidance algorithm, on the other hand, is not influenced by scale and could be applied to almost any kind of power-kite system.

- Real-time optimization: The RTO algorithm is readily applicable to ship-towing and fixed-length tether crosswind electricity generation, but would need to be significantly extended to handle pumping-cycle electricity generation. The MA framework itself is very general and has also been applied to a variety of chemical processes. However, the particular formulation of the optimization problem (the choice of the cost and constraint functions and of the decision variables) must be adapted to each specific problem. For example, in this paper we focused on line-tension maximization, but for a powerful system operating in strong winds, it may sometimes be more important to keep line-tension below a safe limit. Max- 
imization of thrust (the component of line tension in a desired direction) for ship-towing is very similar to the maximization of line tension dealt with in this article. Only a minor change would need to be made to the definition of the cost function and the same decision variables (width and height of the reference path) could be used. The center point of the reference path would be determined by the direction of travel, and when this makes a significant angle with the apparent wind, it might be useful to introduce a further decision variable to represent the asymmetry of the figure-of-eight path.

In fixed-length-tether crosswind kite power with turbines on the wing, instantaneous electricity production is almost proportional to tether tension, and so the optimization algorithm from this paper could be applied with little modification. The reference path should be centered directly downwind, either using wind measurements or using the approach described in [28].

Pumping-cycle electricity generation is unfortunately a little trickier to deal with, as due to the variation in the tether length, each figure-of-eight will be subject to wind at different altitudes. The cost function could be the average electricity produced over an entire pumping cycle, and the decision variables could be the height and width relative to the tether length and either the tether tension or the tether reel-out speed.

Many additions and improvements to this work could be envisaged. In terms of modeling, the law linking a decrease in the lift-to-drag ratio with steering deflections should be more comprehensively validated on a number of kites in varying wind conditions, as we cannot currently guarantee that the relationship is generally valid. In terms of control, if increased path-following accuracy is required, there is no doubt that a well-designed feed-forward signal could improve the velocity control loop. As the reference path is repetitive, another promising option would be to use run-to-run control, such as iterative learning control [45]. The guidance algorithm could be generalized to motion on a sphere using geodesic distances and angles, following the approach in [15]. At the expense of some mathematical abstraction, this framework is advantageous as it does not approximate the quarter-sphere upon which the kite flies using a plane, which results in a certain distortion.

Perhaps the most promising avenue is pitch control, using the kite's second degree of freedom. Up until now, controller development has focused on the "steering" input, which is usually the difference between the lengths of the rear lines. However, the kite's pitch angle relative to the lines can also be adjusted, usually by changing the length of the front line relative to that of the rear lines. This additional degree of freedom is typically used for manual kite control; it directly influences the kite's angle of attack, which has a strong effect on the line tension, the kite's speed, and the kite's turning behavior. A first step in exploiting this degree of freedom would be to establish experimentally validated models describing the influence of the kite's angle of attack, which are currently not available.

In terms of real-time optimization, this study has really only scratched the surface and there is much room for improvement Wind measurements could certainly be used beneficially. If the influence of wind speed and direction on the line tension can be computed using a model, and subtracted from the observed variations, then the line-tension variations due to path changes alone can be more easily detected. At the optimisation level, noise was dealt with using a very basic averaging approach in this study - a far more efficient way to reduce noise would be to use a properly designed low-pass filter.

\section{ACKNOWLEDGMENT}

The authors would like to thank Nikitas Rontsis, Ioannis Lymperopoulos, Jose Afonso, Yannick Poffet, Damien Benoit, Paul Bertusi and Enea Martinoli for their contributions to this project. We would also like to thank Corey Houle, Rolf Luschinger and the entire Swiss Kite Power group for many years of open and inspiring collaboration.

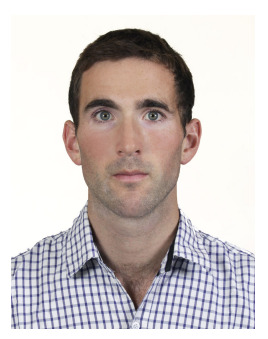

Sean Costello finished a PhD at EPFL in Lausanne, Switzerland in 2015. His research areas are control systems, signal processing, robotics and optimization. He is currently a Control Systems Engineer with Leica Geosystems in Switzerland.

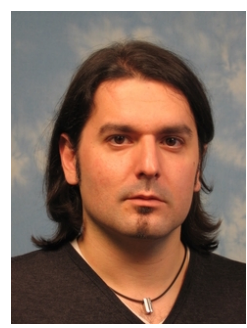

Grégory François received a PhD degree in Process Systems Engineering from EPFL Lausanne, Switzerland in 2004 and an Habilitation Thesis also in Process Systems Engineering from the University of Lorraine, France, in 2014. His research interests include modeling, control, optimal control and optimization of static and dynamical systems, with application to a wide range of systems including chemical, polymerization, energy and fuel-cell systems.

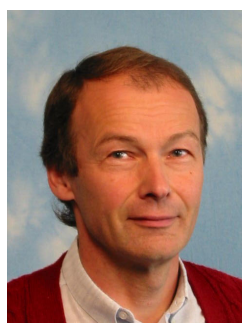

Dominique Bonvin is Director of the Automatic Control Laboratory of EPFL in Lausanne, Switzerland. He received his Diploma in Chemical Engineering from ETH Zurich and his Ph.D. degree from the University of California, Santa Barbara. He served as Dean of Bachelor and Master studies at EPFL between 2004 and 2011. His research interests include modeling, identification and optimization of dynamical systems. 


\section{REFERENCES}

[1] C. L. Archer and M. Z. Jacobson, "Evaluation of global wind power," J. Geophysical Research: Atmospheres, vol. 110, no. D12, 2005.

[2] B. W. Roberts, D. H. Shepard, K. Caldeira, M. E. Cannon, D. G. Eccles, A. J. Grenier, and J. F. Freidin, "Harnessing high-altitude wind power," IEEE Trans. on Energy Conversion, vol. 22, no. 1, pp. 136-144, 2007.

[3] C. L. Archer, "An introduction to meteorology for airborne wind energy," in Airborne Wind Energy, U. Ahrens, M. Diehl, and R. Schmehl, Eds. Berlin: Springer, 2013, pp. 81-94.

[4] G. P. Van den Berg, "Wind gradient statistics up to $200 \mathrm{~m}$ altitude over flat ground," in Proc. $1^{\text {st }}$ International Meeting on Wind Turbine Noise, 2005.

[5] M. Erhard and H. Strauch, "Control of towing kites for seagoing vessels," IEEE Trans. on Control Systems Tech., vol. 21, no. 5, pp. 16291640, 2013.

[6] U. Ahrens, M. Diehl, and R. Schmehl, Eds., Airborne Wind Energy. Berlin: Springer, 2013.

[7] L. Fagiano and M. Milanese, "Airborne wind energy: An overview," in Proc. American Control Conference (ACC), 2012, pp. 3132-3143.

[8] M. L. Loyd, "Crosswind kite power," J. Energy, vol. 4, no. 3, pp. 106111, May 1980.

[9] M. Diehl, "Real-time optimization for large-scale nonlinear processes," Ph.D. dissertation, Ruprecht-Karls-Universität Heidelberg, 2001

[10] A. Ilzhöfer, B. Houska, and M. Diehl, "Nonlinear MPC of kites under varying wind conditions for a new class of large-scale wind power generators," Int. J. Robust and Nonlinear Control, vol. 17, no. 17, pp. 1590-1599, 2007.

[11] M. Canale, L. Fagiano, and M. Milanese, "High altitude wind energy generation using controlled power kites," IEEE Trans. on Control Systems Tech., vol. 18, no. 2, pp. 279-293, 2010.

[12] J. Breukels, R. Schmehl, and W. Ockels, "Aeroelastic simulation of flexible membrane wings based on multibody system dynamics," in Airborne Wind Energy, U. Ahrens, M. Diehl, and R. Schmehl, Eds. Berlin: Springer, 2013, pp. 287-305.

[13] F. Gohl and R. H. Luchsinger, "Simulation based wing design for kite power," in Airborne Wind Energy, U. Ahrens, M. Diehl, and R. Schmehl, Eds. Berlin: Springer, 2013, pp. 325-338.

[14] M. Vukov, S. Gros, G. Horn, G. Frison, K. Geebelen, J. Jørgensen, J. Swevers, and M. Diehl, "Real-time nonlinear MPC and MHE for a large-scale mechatronic application," Control Eng. Practice, vol. 45, pp. 64-78, 2015.

[15] J. Baayen and W. Ockels, "Tracking control with adaption of kites," Control Theory App., IET, vol. 6, no. 2, pp. 182-191, 2012

[16] L. Fagiano, A. U. Zgraggen, M. Morari, and M. Khammash, "Automatic crosswind flight of tethered wings for airborne wind energy: Modeling, control design, and experimental results," IEEE Trans. on Control Systems Tech., vol. 22, no. 4, pp. 1433-1447, 2014.

[17] A. U. Zgraggen, L. Fagiano, and M. Morari, "Automatic retraction and full-cycle operation for a class of airborne wind energy generators," IEEE Trans. on Control Systems Tech., vol. 24, no. 2, pp. 594-608, 2016.

[18] C. Jehle and R. Schmehl, "Applied tracking control for kite power systems," J. Guidance, Control, and Dynamics, vol. 37, no. 4, pp. 12111222, 2014.

[19] R. Ruiterkamp and S. Sieberling, "Description and preliminary test results of a six degrees of freedom rigid wing pumping system," in Airborne Wind Energy, U. Ahrens, M. Diehl, and R. Schmehl, Eds. Berlin: Springer, 2013, pp. 443-458.

[20] A. U. Zgraggen, L. Fagiano, and M. Morari, "On real-time optimization of airborne wind energy generators," in Proc. 52 ${ }^{\text {nd }}$ IEEE Conference on Decision and Control (CDC), Florence, 2013, pp. 385-390.

[21] B. Houska and M. Diehl, "Optimal control of towing kites," in Proc. 45th IEEE Conference on Decision and Control (CDC), 2006, pp. 26932697.

[22] — "Optimal control for power generating kites," in Proc. 9th European Control Conference, 2007, pp. 3560-3567.

[23] P. Williams, B. Lansdorp, and W. Ockesl, "Optimal crosswind towing and power generation with tethered kites," J. Guidance, Control, and Dynamics, vol. 31, no. 1, pp. 81-93, 2008.

[24] I. Argatov and R. Silvennoinen, "Energy conversion efficiency of the pumping kite wind generator," Renewable Energy, vol. 35, no. 5, pp. 1052-1060, 2010

[25] G. M. Dadd, D. A. Hudson, and R. A. Shenoi, "Determination of kite forces using three-dimensional flight trajectories for ship propulsion," Renewable Energy, vol. 36, no. 10, pp. 2667-2678, 2011.
[26] M. Erhard, G. Horn, and M. Diehl, "A quaternion-based model for optimal control of the skysails airborne wind energy system," arXiv preprint arXiv:1508.05494, 2015

[27] G. Horn, S. Gros, and M. Diehl, "Numerical trajectory optimization for airborne wind energy systems described by high fidelity aircraft models," in Airborne Wind Energy, U. Ahrens, M. Diehl, and R. Schmehl, Eds. Berlin: Springer, 2013, pp. 205-218.

[28] A. U. Zgraggen, L. Fagiano, and M. Morari, "Real-time optimization and adaptation of the crosswind flight of tethered wings for airborne wind energy," IEEE Trans. on Control Systems Tech., vol. 23, no. 2, pp. 434-448, 2015

[29] S. Costello, "Real-time optimization via directional modifier adaptation, with application to kite control," Ph.D. dissertation, \# 6571, EPFL, Lausanne, 2015.

[30] S. Costello, G. François, and D. Bonvin, "Directional real-time optimization applied to a kite-control simulation benchmark," in European Control Conference, 2015, pp. 1594-1601.

[31] G. M. Dadd, D. A. Hudson, and R. A. Shenoi, "Comparison of two kite force models with experiment," J. of Aircraft, vol. 47, pp. 212-224, 2010.

[32] J. Breukels, "An engineering methodology for kite design," Ph.D. dissertation, Delft Universtiy of Technology, 2010.

[33] M. Erhard and H. Strauch, "Theory and experimental validation of a simple comprehensible model of tethered kite dynamics used for controller design," in Airborne Wind Energy, U. Ahrens, M. Diehl, and R. Schmehl, Eds. Berlin: Springer, 2013, pp. 141-165.

[34] S. Gros and M. Diehl, "Modeling of airborne wind energy systems in natural coordinates," in Airborne Wind Energy, U. Ahrens, M. Diehl, and R. Schmehl, Eds. Berlin: Springer, 2013, pp. 181-203.

[35] A. Bosch, R. Schmehl, P. Tiso, and D. Rixen, "Nonlinear aeroelasticity, flight dynamics and control of a flexible membrane traction kite," in Airborne Wind Energy, U. Ahrens, M. Diehl, and R. Schmehl, Eds. Berlin: Springer, 2013, pp. 307-323.

[36] X. Paulig, M. Bungart, and B. Specht, "Conceptual design of textile kites considering overall system performance," in Airborne Wind Energy, U. Ahrens, M. Diehl, and R. Schmehl, Eds. Berlin: Springer, 2013, pp. $547-562$.

[37] T. A. Wood, H. Hesse, A. U. Zgraggen, and R. S. Smith, "Modelbased flight path planning and tracking for tethered wings," in 54th IEEE Conference on Decision and Control (CDC), 2015, pp. 6712-6717.

[38] Y. Kanayama, Y. Kimura, F. Miyazaki, and T. Noguchi, "A stable tracking control method for an autonomous mobile robot," in Proceedings of the IEEE International Conference on Robotics and Automation, vol. 1, 1990, pp. 384-389.

[39] A. Bosch, R. Schmehl, P. Tiso, and D. Rixen, "Dynamic nonlinear aeroelastic model of a kite for power generation," Journal of Guidance, Control, and Dynamics, vol. 37, no. 5, pp. 1426-1436, 2014.

[40] N. Rontsis, S. Costello, I. Lymperopoulos, and C. N. Jones, "Improved path following for kites with input delay compensation," in 54th IEEE Conference on Decision and Control (CDC), 2015, pp. 656-663.

[41] T. A. Wood, H. Hesse, A. U. Zgraggen, and R. S. Smith, "Modelbased identification and control of the velocity vector orientation for autonomous kites," in American Control Conference (ACC), 2015, pp. 2377-2382.

[42] D. Nelson, D. Barber, T. McLain, and R. Beard, "Vector field path following for miniature air vehicles," IEEE Trans. on Robotics, vol. 23, no. 3, pp. 519-529, 2007

[43] A. Marchetti, B. Chachuat, and D. Bonvin, "Modifier-adaptation methodology for real-time optimization," Ind. Eng. Chem. Res., vol. 48, no. 13 , pp. $6022-6033,2009$.

[44] S. Costello, G. François, and D. Bonvin, "A directional modifier adaptation algorithm for real-time optimization." $J$. Process Control, vol. 39, pp. 64-76, 2016. [Online]. Available: http://infoscience.epfl.ch/record/202628

[45] K. L. Moore, Iterative Learning Control for Deterministic Systems. Springer-Verlag, Advances in Industrial Control, London, 1993. 